\title{
ERS/ESTS/EACTS/ESTRO guidelines for the management of malignant pleural mesothelioma
}

\author{
Arnaud Scherpereel ${ }^{1,2}$, Isabelle Opitz ${ }^{3}$, Thierry Berghmans ${ }^{4}$, Ioannis Psallidas $^{5}$, \\ Markus Glatzer ${ }^{6}$, David Rigau ${ }^{7}$, Philippe Astoul $\mathbb{1}^{8}$, Servet Bölükbas ${ }^{9}$, \\ Jeanette Boyd $^{10}$, Johan Coolen ${ }^{11}$, Charlotte De Bondt ${ }^{12}$, Dirk De Ruysscher ${ }^{13}$, \\ Valerie Durieux (10 ${ }^{14}$, Corinne Faivre-Finn ${ }^{15}$, Dean Fennell ${ }^{16}$, \\ Francoise Galateau-Salle ${ }^{17}$, Laurent Greillier (10 ${ }^{18}$, Mir Ali Hoda ${ }^{19}$, \\ Walter Klepetko ${ }^{19}$, Aude Lacourt ${ }^{20}$, Phil McElnay ${ }^{21}$, Nick A. Maskell ${ }^{22}$, \\ Luciano Mutti ${ }^{23}$, Jean-Claude Pairon $\mathbb{1 0}^{24}$, Paul Van Schil ${ }^{25}$, Jan P. van Meerbeeck ${ }^{12}$, \\ David Waller ${ }^{26}$, Walter Weder ${ }^{3}$, Giuseppe Cardillo ${ }^{27}$ and Paul Martin Putora ${ }^{6,28}$
}

@ERSpublications

A European expert task force proposes updated and practical guidelines on routine management of malignant pleural mesothelioma, after a systematic review of the 2009-2018 literature (GRADE), including new promising therapies and strategies http://bit.ly/38876ta

Cite this article as: Scherpereel A, Opitz I, Berghmans T, et al. ERS/ESTS/EACTS/ESTRO guidelines for the management of malignant pleural mesothelioma. Eur Respir J 2020; 55: 1900953 [https://doi.org/ 10.1183/13993003.00953-2019].

ABSTRACT The European Respiratory Society (ERS)/European Society of Thoracic Surgeons (ESTS)/ European Association for Cardio-Thoracic Surgery (EACTS)/European Society for Radiotherapy and Oncology (ESTRO) task force brought together experts to update previous 2009 ERS/ESTS guidelines on management of malignant pleural mesothelioma (MPM), a rare cancer with globally poor outcome, after a systematic review of the 2009-2018 literature. The evidence was appraised using the Grading of Recommendations, Assessment, Development and Evaluation approach. The evidence syntheses were discussed and recommendations formulated by this multidisciplinary group of experts. Diagnosis: pleural biopsies remain the gold standard to confirm the diagnosis, usually obtained by thoracoscopy but occasionally via image-guided percutaneous needle biopsy in cases of pleural symphysis or poor performance status. Pathology: standard staining procedures are insufficient in $\sim 10 \%$ of cases, justifying the use of specific markers, including $B A P-1$ and $C D K N 2 A$ ( $p 16$ ) for the separation of atypical mesothelial proliferation from MPM. Staging: in the absence of a uniform, robust and validated staging system, we advise using the most recent 2016 8th TNM (tumour, node, metastasis) classification, with an algorithm for pre-therapeutic assessment. Monitoring: patient's performance status, histological subtype and tumour volume are the main prognostic factors of clinical importance in routine MPM management. Other potential parameters should be recorded at baseline and reported in clinical trials. Treatment: (chemo)therapy has limited efficacy in MPM patients and only selected patients are candidates for radical surgery. New promising targeted therapies, immunotherapies and strategies have been reviewed. Because of limited data on the best combination treatment, we emphasise that patients who are considered candidates for a multimodal approach, including radical surgery, should be treated as part of clinical trials in MPM-dedicated centres.

This article has supplementary material available from erj.ersjournals.com

Received: 12 May 2019 | Accepted after revision: 17 Oct 2019

The article has been co-published with permission in the European Respiratory Journal and the European Journal of Cardio-Thoracic Surgery. All rights reserved in respect of European Respiratory Journal, ( E) European Respiratory Society 2020 and European Journal of Cardio-Thoracic Surgery, (c) European Association for Cardio-Thoracic Surgery 2020. The articles are identical except for minor stylistic and spelling differences in keeping with each journal's style. Either citation can be used when citing this article. 


\section{Introduction}

Malignant pleural mesothelioma (MPM) is a rare tumour that has become a world health issue due to its poor prognosis and its increasing incidence, largely due to prior asbestos exposure. However, there has been a remarkable improvement of the knowledge of MPM pathogenesis in recent years, leading to new potential drugs and strategies $[1,2]$. Moreover, recent results from trials with multimodal treatment or innovative drugs such as targeted therapies or immunotherapies have brought new hope for MPM patients [3].

Optimal treatment in MPM has not previously been well defined and recent informative guidelines from the British Thoracic Society [4], the American Society of Clinical Oncology [5], the National Comprehensive Cancer Network (NCCN) [6] and the European Society for Medical Oncology [7] have reviewed similar published evidence and came to different conclusions and recommendations. This task force was conducted by the European Respiratory Society (ERS) in collaboration with the European Society of Thoracic Surgeons (ESTS), the European Association for Cardio-Thoracic Surgery (EACTS) and the European Society for Radiotherapy and Oncology (ESTRO). It brought together experts on mesothelioma from different scientific societies to update the previous recommendations [8], with the aim of providing clinicians with a clear, concise and up-to-date statement on MPM management.

\section{Methods}

The purpose of these guidelines is to update the previous ERS/ESTS clinical practice guidelines for the management of MPM [8] and provide evidence-based recommendations for specialist care clinicians who want to offer patients a therapeutic approach based on radiotherapy, surgery, (chemo)therapy (first-line and salvage) or a combination of these modalities. Epidemiology, aetiology, biomarkers and screening of asbestos-exposed populations, clinical and pathological diagnosis and staging as well as treatment allocation have been summarised narratively and research priorities have been issued.

This current joint ERS/ESTS/EACTS/ESTRO task force was co-chaired by AS, IO, PMP and GC and included 28 clinicians with experience in several disciplines of MPM management and research and one European Lung Foundation representative (JB). One methodologist (DR) ensured that all the methodological requirements were met. The co-chairs and task force members discussed the evidence and formulated the recommendations; the methodologist did not participate in the development of recommendations. All panel members were required to disclose their conflicts of interest.

A first literature search was performed in November 2016 using the Ovid MEDLINE system. This research was performed by a scientific librarian (VD), experienced in searching for medical and scientific publications, and by physicians, experts in the treatment of thoracic neoplasms and trained in

Affiliations: ${ }^{1}$ Pulmonary and Thoracic Oncology, Univ. Lille, CHU Lille, INSERM U1189, OncoThAl, Lille, France. ${ }^{2}$ French National Network of Clinical Expert Centers for Malignant Pleural Mesothelioma Management (Mesoclin), Lille, France. ${ }^{3}$ Dept of Thoracic Surgery, University Hospital Zurich, Zurich, Switzerland. ${ }^{4}$ Thoracic Oncology Clinic, Institut Jules Bordet, Brussels, Belgium. ${ }^{5}$ Oxford Centre for Respiratory Medicine, Oxford University Hospitals NHS Foundation Trust, Oxford, UK. ${ }^{6}$ Dept of Radiation Oncology, Kantonsspital St Gallen, St Gallen, Switzerland. ${ }^{7}$ Iberoamerican Cochrane Center, Barcelona, Spain. ${ }^{8}$ Dept of Thoracic Oncology, Pleural Diseases and Interventional Pulmonology, Hôpital Nord, Aix-Marseille University, Marseille, France. ${ }^{9}$ Dept of Thoracic Surgery, Evang, Kliniken Essen-Mitte, Essen, Germany. ${ }^{10}$ European Lung Foundation, Sheffield, UK. ${ }^{11}$ Dept of Imaging and Pathology, KU Leuven, Leuven, Belgium. ${ }^{12}$ Dept of Pulmonology and Thoracic Oncology, Antwerp University and Antwerp University Hospital, Antwerp, Belgium. ${ }^{13}$ Dept of Radiation Oncology (Maastro Clinic), Maastricht University Medical Center+, GROW Research Institute, Maastricht, The Netherlands. ${ }^{14}$ Bibliothèque des Sciences de la Santé, Université libre de Bruxelles (ULB), Brussels, Belgium. ${ }^{15}$ The Christie NHS Foundation Trust, The University of Manchester, Manchester, UK. ${ }^{16}$ Leicester Cancer Research Centre, University of Leicester and University of Leicester Hospitals NHS Trust, Leicester, UK. ${ }^{17}$ National Reference Center for Pleural Malignant Mesothelioma and Rare Peritoneal Tumors MESOPATH, Dept of Biopathology, Centre Leon Berard, Lyon, France. ${ }^{18}$ Aix Marseille University, Assistance Publique Hôpitaux de Marseille, Inserm UMR1068, CNRS UMR7258, Dept of Multidisciplinary Oncology and Therapeutic Innovations, Marseille, France. ${ }^{19}$ Dept of Thoracic Surgery, Medical University of Vienna, Vienna, Austria. ${ }^{20}$ Univ. Bordeaux, INSERM, Bordeaux Population Health Research Center, team EPICENE, UMR 1219, Bordeaux, France. ${ }^{21}$ Newcastle University, Newcastle upon Tyne, UK. ${ }^{22}$ Academic Respiratory Unit, Bristol Medical School, University of Bristol, Bristol, UK. ${ }^{23}$ Teaching Hosp. Vercelli/Gruppo Italiano Mesotelioma, Italy. ${ }^{24}$ INSERM U955, Equipe 4, Université Paris-Est Créteil, and Service de Pathologies professionnelles et de l'Environnement, Institut Santé-Travail Paris-Est, CHI Créteil, Créteil, France. ${ }^{25}$ Dept Thoracic and Vascular Surgery, Antwerp University and Antwerp University Hospital, Antwerp, Belgium. ${ }^{26}$ Barts Thorax Centre, St Bartholomew's Hospital, London, UK. ${ }^{27}$ Unit of Thoracic Surgery, Azienda Ospedaliera San Camillo Forlanini, Rome, Italy. ${ }^{28}$ Dept of Radiation Oncology, University of Bern, Bern, Switzerland.

Correspondence: Arnaud Scherpereel, Pulmonary and Thoracic Oncology Dept, CHU de Lille, F-59037 Lille Cedex, France. E-mail: arnaud.scherpereeldachru-lille.fr 
evidence-based medicine. The Ovid MEDLINE database was searched using the OvidSP interface. The "Population, Intervention, Comparison, Outcome" (PICO) questions model for clinical questions was used to identify the concepts included in the questions, as shown in the supplementary material [9]. The corresponding search criteria were translated into Medical Subject Headings (MeSH) terms, free-text keywords and name of substances or interventions (supplementary material). Results were limited to articles published from 2009 to the present. It was a search strategy decision to limit the start of the search to 2009, after the previous ERS/ESTS guidelines, to restrict it to pertinent citations, as a systematic search of the literature up to 2008 was conducted by the previous task force. Citations were exported from MEDLINE into reference manager databases (EndNote) to allow the removal of duplicates and to facilitate the selection process performed by reviewers. All articles retrieved by the librarian were selected for their eligibility by two authors based on the title and abstract, and the final selection was performed by reading the full publication and its inclusion was decided by consensus. This search was supplemented by screening the references of the selected articles and other literature known to the experts.

An update of the literature was performed on January 2019 in order to capture randomised clinical trials relevant to the clinical questions. Supplementary figure S1 shows a flow chart of the literature search.

We used the Grading of Recommendations, Assessment, Development and Evaluation (GRADE) approach to appraise the quality of evidence and to formulate, write and grade most recommendations. GRADEpro Guideline Development Tool software (McMaster University, 2015; developed by Evidence Prime, Hamilton, ON, Canada) was used to develop evidence profiles that summarised the findings for each outcome and the rationale for the quality of evidence appraisal [9].

The evidence profiles were sent to the task force members for review. Using an iterative consensus process conducted face to face, via teleconference and via email, recommendations were formulated on the basis of the following considerations: the balance of desirable (benefits) and undesirable consequences (burden, adverse effects and cost) of the intervention, the quality of evidence, acceptability and feasibility.

A strong recommendation for an intervention indicates that most well-informed patients would choose the intervention, whereas a conditional recommendation for an intervention indicates that well-informed patients may make different choices.

Thus, based on an extensive search of the literature (2009-2019) on MPM, the authors answered several questions on this cancer, to update previous European guidelines [8], including the following PICO questions:

\section{Surgery}

Should partial pleurectomy, compared to talc pleurodesis, be used as a palliative procedure in patients with symptomatic MPM?

Should "radical surgery" (including extrapleural pneumonectomy or pleurectomy/decortication) be used in patients with MPM?

\section{Radiotherapy}

Should radiotherapy be used for pain relief in patients with MPM?

Should radiotherapy be used to prevent procedure-tract metastases (drain site parietal seeding) in patients with MPM?

Should adjuvant postoperative radiotherapy be used in patients with MPM?

Medical treatment

Should first-line (chemo)therapy consisting of platinum alone or in combination with pemetrexed be used in patients with MPM?

Should bevacizumab be added to first-line standard (chemo)therapy in patients with MPM?

Should targeted therapies be added to first-line standard (chemo)therapy in patients with MPM?

Should immunotherapy be used as salvage therapy in patients with MPM who failed first-line standard (chemo)therapy?

Multimodality

Should a multimodal therapy approach (combining more than one method of cancer treatment: surgery, (chemo)therapy, radiation therapy) compared to (chemo)therapy alone be used in patients with MPM?

\section{Epidemiology of mesothelioma}

Incidence trend and predictions

From publications investigating the incidence trend at the world level, it appears that there is a lack of data regarding mesothelioma incidence and/or mortality for a large part of the world population [10-12] and especially for countries still using asbestos, such as in Eastern Europe, Asia, South America and most of Africa [13]. From available data, large disparities in mesothelioma incidence/mortality rates and trends are noticeable from country to country (supplementary table S1) [10-12, 14-43]. 
The pattern of mesothelioma incidence is highly correlated with the pattern of asbestos importation and use $[14,44]$ with a delay of $\sim 40$ years due to the long latency period. It has been estimated that the incidence peak in Western Europe will be reached around 2020, and epidemiological data support these predictions [45]. Lower incidence rates in some parts of Asia and Central or Eastern European countries may be related to a poorer quality of data regarding diagnostic certification and registration [46] and a higher mortality from other causes. Besides, due to the long latency period, the epidemic of mesothelioma in those countries is likely to be at its beginning $[13,14]$.

The task force experts consider essential that all countries set up permanent epidemiological surveillance systems based on the exhaustive registration of mesothelioma cases at a national level.

\section{Mesothelioma aetiology}

Asbestos exposure

Asbestos is the principal aetiological agent of MPM. The term asbestos refers to six silicate minerals which are able to form very thin fibres, divided between the serpentine group (chrysotile) and the amphibole group of minerals (crocidolite, amosite, anthophyllite, tremolite and actinolite). Chrysotile is less biopersistent in the lungs than amphiboles. Chrysotile, amosite and crocidolite have all been widely used for industrial purposes.

To date, there are no new data questioning the previous guidelines [8]: 1) a dose-response relationship between asbestos exposure and mesothelioma occurrence has been demonstrated [47]; 2) however, it is still impossible to define a threshold of cumulative exposure below which there is no increased risk, implying that all exposed individuals are constituting a population at risk; and 3) the mean (range) latency of MPM following asbestos exposure is 40 (15-67) years [48].

Occupational asbestos exposure accounts for $>80 \%$ of cases in males (supplementary table S2) [49-52] and the differences in attributable risk between males and females is probably due to household [53, 54] or environmental exposure (supplementary table S3) [51, 52, 55-74].

\section{Exposure to other elongated mineral particles}

Other elongated mineral particles such as erionite or fluoro-edenite may be involved in the aetiology of malignant mesothelioma (supplementary table S4) [75-94], with potential environmental exposure in various countries, such as Turkey, USA and Mexico [95-97].

From the available literature, occupational exposure to refractory ceramic fibres does not seem to be associated with the occurrence of MPM [88, 89]. However, some studies have raised the hypothesis of a synergistic effect between co-exposure to asbestos and other synthetic fibres, namely refractory ceramic fibres or mineral wool fibres [51, 98-100].

In 2014, in the absence of human data, multiwalled carbon nanotubes (MWCNT)-7 was classified by the International Agency for Research on Cancer (IARC) as possibly carcinogenic to humans (group 2B), while other sorts of carbon nanotubes were not classifiable as to their carcinogenicity to humans (group 3) [90, 94]. Recent experimental studies demonstrated the induction of MPM following intratracheal instillation of MWCNT into rat lungs [101, 102].

\section{Genetic predisposition}

Studies of familial aggregation of mesothelioma cases have reported an increased risk for subjects having parents and siblings diagnosed with mesothelioma [103-105]. Those observations led to the identification of a genetic component involved in the increased risk of mesothelioma in those families [106-112], namely a germline mutation of the BRCA1-associated protein (BAP)-1 gene, a tumour suppressor gene involved in the modulation of transcription and DNA repair. Other studies have attempted to identify new loci that might be associated with mesothelioma [111, 113-120].

A significant proportion of patients with malignant mesothelioma carry germline mutations in cancer susceptibility genes, especially those with peritoneal mesothelioma, minimal asbestos exposure, young age and a second cancer diagnosis [121, 122].

These data support clinical germline genetic testing for selected patients with malignant mesothelioma and provide a rationale for additional investigation of genetic pathways in malignant mesothelioma.

\section{Other risk factors}

Ionising radiation (mainly therapeutic radiation) is a risk factor for mesothelioma [123, 124], although it accounts for a small proportion of mesothelioma cases relative to asbestos exposure [13]. 
There were some controversies regarding the implication of the simian virus 40 (SV40) in MPM pathogenesis. In 2014, the IARC considered that SV40 could not be classified as carcinogenic to humans (group 3) [125]. It should be noted that tobacco smoking is not a risk factor for MPM.

The task force experts consider that national and international authorities must take an active role to achieve a complete and definitive ban of asbestos use worldwide, and to promote a close watch of other potential risk factors for MPM.

\section{Biomarkers and screening in asbestos-exposed populations}

Screening for MPM would raise many issues about the target population, the most efficient tool(s) to use, and, primarily, the rationale of such screening for a quite rare cancer.

\section{Pleural plaques and MPM}

Based on several consensus statements of an increased prevalence of pleural plaques among mesothelioma cases compared to non-mesothelioma subjects, the hypothesis of an association between pleural plaques and MPM has been raised [126-128]. However, since pleural plaques are considered a marker of asbestos exposure, it is not surprising to find such association and it is challenging to estimate the independent association between pleural plaques and MPM, considering that asbestos exposure is a strong confounder in this relationship. While most studies were based on chest radiograph detection of pleural plaques, recently, a positive and significant association between pleural plaques and MPM was found, detected using computed tomography (CT) scanning, while accounting for occupational asbestos exposure [129]. However, some authors have suggested that it cannot be ruled out that pleural plaques are only a marker of asbestos exposure [128].

Pleural plaques are likely to be a simple marker of previous asbestos exposure; the task force experts consider that no invasive diagnostic procedure is justified due to their presence. However, CT scans could detect (benign) asbestos-related lung diseases in exposed subjects, which may justify compensation according to national rules, but which may also be a marker of increased risk of MPM.

Research priority: the relationship between pleural plaques and MPM should be ascertained in large international epidemiological studies. The effectiveness of CT screening in the asbestos-exposed population should be determined in well-designed clinical trials.

\section{(Diagnostic) biomarkers}

Several blood biomarkers have been proposed for MPM screening, diagnosis, prognosis or follow-up during treatment. Results of biomarkers applied in populations for diagnosis purposes are summarised in supplementary table S5 [130-152]. The performance of these markers tested alone or in combination have been evaluated and reviews published [153-157]. A meta-analysis on the diagnostic value of soluble mesothelin in $>4000$ patients estimated sensitivity and specificity at $47 \%$ and $95 \%$, respectively [135]. A few prospective studies conducted in subjects previously exposed to asbestos (supplementary tables S6 and S7) failed to demonstrate any value of serum mesothelin as a screening tool in these populations [158-164]. Simulations of real-life use of biomarkers (supplementary table S8) found a very high number of false-positive cases, even in populations highly exposed to asbestos. The role of mesothelin and other biomarkers for monitoring the response to antitumour treatment are currently being evaluated in a number of centres.

Research priority: routine determination of previously proposed biomarkers in MPM have no current validated role in diagnosis, prognosis or clinical follow-up (disease monitoring). Thus, further research into the role of biomarkers in these goals is required and highly encouraged.

\section{Methods of assessing asbestos exposure}

No significant change was found since the 2009 ERS/ESTS guidelines [8].

\section{MPM compensation}

As occupational asbestos exposure is strongly associated with the occurrence of mesothelioma, some countries have set up compensation programmes, i.e. recognition of MPM as an occupational disease [18, 165-167] and/or compensation from asbestos victims' funds [167]. An analysis of the literature [18, 165-167] suggests undercompensation for MPM cases.

The task force experts consider that the dissemination of information to clinicians and patients regarding the right to compensation for MPM should be reinforced according to the specific rules applying in each country. 


\section{Diagnosis of MPM}

Clinical manifestations

The following recommendations from the 2009 ERS/ESTS guidelines are still valid in 2019 without any change according to the 2009-2017 literature [8].

The clinical manifestations of MPM are usually nonspecific and insidious and should not be used alone as diagnostic criteria, even in cases of previous asbestos exposure.

Chest radiography usually shows a unilateral pleural effusion and/or thickening. Chest radiography alone should not be used for the diagnosis of MPM. In addition, chest CT scan is unsuitable for definitive diagnosis of MPM, but diffuse or nodular pleural thickening is suggestive of the disease, especially involving mediastinal pleura. Chest CT scans with intravenous contrast agent (optimised for pleural evaluation) is the modality of choice for initial evaluation of patients with suspected MPM. Positron emission tomography (PET)-CT can be used to provide useful functional information on pleural lesions, if prior talc pleurodesis has not been performed, even if it not specific enough to diagnose MPM routinely. Functional magnetic resonance imaging (MRI) may be considered in these situations and other difficult diagnostic cases. MRI data appear promising, but are yet to be validated prospectively. The imaging modalities are the cornerstone of determining the correct biopsy site.

What is the best pleural biopsy method in suspected cases of mesothelioma?

Thoracoscopic biopsies (performed under local or general anaesthesia) are the gold standard for investigating an undiagnosed pleural effusion where the differential diagnosis includes mesothelioma. However, other biopsy methods are less invasive and may be more appropriate in selected cases. Thus, image-guided cutting-needle biopsies have high diagnostic rates and are particularly useful in patients with pleural thickening without associated pleural effusion, or in frail patients not fit enough for thoracoscopy. In particular, thoracic ultrasonography (TUS) allows the physician and radiologist to perform pleural biopsies more accurately and safely without any radiation exposure.

\section{Blind closed-needle biopsies}

The sensitivity of Abrams biopsies for malignancy is between $27 \%$ and $60 \%$ [168-172], being much lower for mesothelioma diagnosis. In the largest review of 2893 Abrams samples, diagnostic yield was only 57\% for malignant disease [171]. Because of its poor yield, its use is diminishing in most developed countries and it cannot be recommended as a first-line investigation in this setting.

Image-guided pleural biopsy

The sensitivity of image-guided biopsy has been reported in a number of observational series, with both ultrasound- and CT-guided biopsies being superior to blind pleural biopsy [173, 174]. A prospective randomised trial comparing CT-guided cutting-needle biopsies with Abrams biopsy demonstrated that cutting-needle biopsies were $40 \%$ more sensitive at diagnosing malignancy [175]. The yield from CT-guided biopsy was $87 \%$, compared with $47 \%$ for Abrams biopsies $(p=0.02)$, with the added benefit of fewer passes of the needle in the image-guided group. This is important in cases of suspected mesothelioma where tumour seeding can occur along biopsy tracks.

A recent publication suggests that physician-led, ultrasound-guided pleural biopsy is effective, both as a planned procedure in patients not suitable for thoracoscopy, and as a secondary "on-the-table" option if thoracoscopy fails [176]. Diagnoses were obtained in 47 (94\%) out of 50 patients. Out of 15 patients with a final diagnosis of malignancy, ultrasound-guided biopsy provided diagnostic material in 13 (87\%).

Video-assisted thoracic surgery and medical thoracoscopy

Video-assisted thoracic surgery (VATS) and medical thoracoscopy plays an important role in the diagnosis of MPM. As well as securing a pathological diagnosis [177], it also allows evacuation of symptomatic pleural effusion and pleurodesis using talc poudrage [178]. In addition, it permits the assessment of the pleural cavity for staging purposes, in particular the assessment of visceral pleural and diaphragmatic pleural invasion, which are important prognostic factors [179].

\section{Local-anaesthetic thoracoscopy or medical thoracoscopy}

The diagnostic yield of medical thoracoscopy for pleural malignancy is high. Pooled results from 1369 patients in 22 case series showed an overall diagnostic sensitivity of 92\% [180]. Medical thoracoscopy has been shown to be more successful at diagnosing malignancy than blind or image-guided Abrams biopsies [181-183], and had a higher diagnostic yield than CT-guided cutting-needle biopsies in one small randomised trial [184]. The complication rates are very low, with analysis of 47 studies including 4756 
patients reporting a mortality rate of $0.34 \%$, major complications in $1.8 \%$ and minor complications in $7.8 \%$ of cases [180].

VATS

VATS pleural biopsies carry a sensitivity of $95 \%$, specificity of $100 \%$ and negative predictive value of $94 \%$. This is similar to medical thoracoscopy, although no randomised trial has directly compared the two procedures. VATS offers the additional benefit of allowing the performance of more invasive surgical interventions, such as lung resection and tumour debulking, at the same time as the diagnostic procedure. It is important to note that VATS can be performed under local anaesthesia on nonintubated patients [185].

Tumour spread at resected previous chest tracts and scars is common and was identified as a negative prognosticator for long-term survival $[186,187]$. Therefore, VATS (or medical thoracoscopy) incisions should be generally in line with possible forthcoming thoracotomy incisions [188]. This allows the resection of VATS (or medical thoracoscopy) tracts at the time of future surgery to avoid tumour recurrence in these areas $[189,190]$.

Open pleural biopsy

Sometimes, due to an obliterated pleural space secondary to locally advanced disease, VATS is not possible. In such cases, a small muscle-sparing incision within an intercostal space (with and without associated partial rib resection) allows for open pleural biopsy. CT- or TUS-guided cutting-needle biopsy is another option in this setting. Therefore, thoracotomy is usually not necessary for the accurate diagnosis of MPM.

\section{Pathology}

The diagnosis of mesothelioma is purely histological, based on an adequate tissue specimen and on international evidence-based comprehensive classification agreed by experts throughout the world. The World Health Organization (WHO) histological classification was updated in 2015 [191]. The development of recommendations for MPM pathology was not considered in the scope of these guidelines, because the European task force experts considered that the recommendations from the International Collaboration on Cancer Reporting and the recent update of the International Mesothelioma Interest Group consensus statement are applicable in this context [192, 193].

Clinical information is required for an accurate diagnosis by the pathologist, because it can influence the initial hypothesis, the processing of the specimen, the procedure of sampling and the ancillary analysis to be performed (immunohistochemistry (IHC), the choice of antibodies, fluorescence in situ hybridisation (FISH) analysis, RNA sequencing, comparative genomic hybridisation array, etc.).

\section{Histopathological specimen examination according to MPM clinical presentation}

As pleural effusion is usually the first clinical sign of MPM, cytology is often the first diagnostic procedure to be performed. However, most effusions are caused by the epithelioid type, since sarcomatoid mesothelioma does not usually shed cells into the serosal cavity [194]. Distinction from benign pleural lesions can be impossible on cytology alone, because subpleural fat tissue invasion, which is the most important criterion for malignancy, is lacking. However, recent tests based on molecular abnormalities can be valuable tools. Cytological suspicion of mesothelioma should be followed by tissue confirmation.

The International Mesothelioma Panel recommended that disease recurrence and metastases can be ascertained on cytology alone [193]. However, according to these latest guidelines, in patients unable to benefit from pleural tissue biopsies, a diagnosis of MPM could be ascertained on pleural effusion cytology alone when using specific ancillary techniques, and be as reliable as tissue biopsy, even if the sensitivity remains lower (30-75\%). Thus, although cytology of pleural effusion is not recommended for obtaining an initial firm diagnosis of MPM, it may be very useful for differentiating MPM from other, more common malignancies, e.g. lung carcinoma. Cytology is more reliable if pleural exudate is preserved in cytoblocks and if ancillary tests (IHC or genetic testing, e.g. p16 deletion in FISH) can be performed [193, 195].

Therefore, as the production of cytoblocks is not a routine procedure in all institutions, the experts would like to highlight the necessity of preparing cytoblocks from pleural effusion samples.

Diagnosis of mesothelioma from fine-needle biopsies is associated with the same diagnostic constraints as pleural cytology, with a low sensitivity $(30 \%)$ [196, 197]. A conclusive diagnosis can only be made if the material is representative of the tumour with sufficient quantity to allow IHC and FISH analysis characterisation in the context of appropriate clinical, radiological and/or surgical findings [198]. 


\section{Macroscopy}

The macroscopic aspect of mesothelioma varies during the natural history of the tumour. Therefore, the topography of the tumour is an important component for pathological staging. A diagnosis of diffuse MPM is more suggestive when the mesothelioma progresses and forms a rind of tumour encasing the lung. Nevertheless, other secondary or primary tumours may have a misleading pseudomesotheliomatous gross characteristic. The type of biopsy may affect the accurate typing and subtyping of diffuse MPM. In addition, it is important to know if the lesion is localised or diffuse, principally because (rare) localised MPM might benefit from surgical resection [194].

\section{Microscopy}

The task force experts consider the 2015 WHO classification reasonable, because it provides a comparative basis for diagnosis, prognosis and therapeutic management of the patient. However, it is well known that some epithelioid mesothelioma subtypes have a better prognosis (papillary, acinar, trabecular), while others have a worse prognosis (solid). Moreover, the presence of particular stromal responses (with abundant myxoid stroma or the rare lymphohistiocytoid variant) also has prognostic value. Some cytological features are associated with a poor outcome (pleomorphic and transitional). The current definition of biphasic mesothelioma requires that $\geqslant 10 \%$ of both epithelioid and sarcomatoid components be present. There is a consensus agreement that if the percentage of sarcomatoid component is $<80 \%$ in the diagnosis of biphasic mesothelioma, it is correlated with a better prognosis. The evaluation of the percentage of the sarcomatoid component is restricted to resected tumours (large surgical specimens) and should not be evaluated on smaller samples [199].

\section{Role of IHC}

IHC enables the separation of different MPM subtypes from other malignancies or pleural metastases, using various sets of antibodies, with a relatively high diagnostic accuracy (supplementary tables S9-S11). In addition to these markers, claudin 4 has recently emerged as one of the most useful markers to separate mesothelioma (claudin 4-negative) from adenocarcinomas (claudin 4-positive) such as breast cancer metastases [193]. Furthermore, sarcomatoid mesothelioma may be cytokeratin-negative in $5 \%$ of cases and in $10 \%$ if heterologous elements are present; in this situation the diagnosis should only be made in the context of appropriate clinical, radiological and/or surgical findings [194].

The three well-defined genetic alterations in diffuse MPM are loss of neurofibromatosis 2 ( Nf2) by mutation or heterozygous or homozygous deletion, observed in $45-50 \%$ of cases; the homozygous deletion of the gene CDKN2A (p16) located on the 9p21 locus, reported in nearly $100 \%$ of sarcomatoid mesothelioma [200]; and loss (absence of nuclear staining when a positive internal control is present on the slide) of BAP-1 (a tumour suppressor gene located on 3p21 locus) by mutation, biallelic deletion or deletion/insertion, detected in $45-100 \%$ of diffuse MPM, mostly epithelioid subtype. While the loss of Nf2 has not proven to be useful in the IHC diagnostic routine [201], BAP-1 loss is a reliable marker on paraffin-embedded tissue and cytoblock section and is associated with a better prognosis. Loss of CDKN2A (p16) detected on formalin-fixed, paraffin-embedded sections as well as on cytoblocks using FISH is associated with a worse prognosis and observed with a sensitivity up to 50\%, being higher in sarcomatoid mesothelioma. The presence of homozygous deletion of the CDKN2A (p16) by FISH analysis is extremely useful, specifically when subpleural fat tissue or lung parenchyma invasion are missing, and favours the diagnosis of malignancy if there is a strong clinical context and radiological evidence of a pleural tumoural process. However, it should be taken into account that BAP-1 loss and p16 are not 100\% specific for mesothelioma.

The loss of BAP-1 expression and/or CDKN2A (p16) homozygous deletion may allow the discrimination of MPM from benign pleural lesions. Given the prognostic and therapeutic significance of BAP-1 loss, BAP-1 may be assessed first by IHC.

Electron microscopy is time- and resource-consuming, and is no more useful with IHC and FISH assays. Finally, freezing pleural tumour tissue is not required routinely, but it may be highly valuable for academic and translational research projects. If so, quality control of the specimen should be performed, and informed consent is needed for ethical biobanking.

\section{Staging and prognosis assessment}

8th TNM revision

The International Association for the Study of Lung Cancer (IASLC) mesothelioma staging project experts have updated their initial findings [202] using prospective data on $>3500$ patients treated both surgically and nonsurgically [203]. Their recommendations [204, 205] will inform the 8th revision of the American Joint Committee on Cancer/Union for International Cancer Control TNM staging system for mesothelioma, summarised here. 
Clinical staging

T stage

T1a (parietal pleura) and T1b (visceral pleura) have been combined into one T1 classification with tumours involving the ipsilateral parietal or visceral pleura only. The T2 classification was used most often due to lung invasion or involvement of fissures. T4 stage was usually due to diffuse chest wall, diaphragm or transmural pericardial invasion. The most common deficiency of clinical staging was the failure to identify occult chest wall or pericardial invasion. In these cases, upstaging was demonstrated subsequently following surgery.

Exploratory analysis suggests that absolute measurement of pleural tumour thickness correlates with survival. When measurements of maximal thickness at upper (apex to inferior margin of aortic arch), middle (between upper and lower) and lower (inferior to left atrium) zones were taken, both the maximum thickness at any level or the sum of the thickness were prognostic. Pleural thickness (maximum or sum) correlated with T stage and nodal positivity [204].

Research priority: prospective data collection about the measurement of tumour thickness or volume is to be encouraged.

\section{$N$ stage}

The IASLC staging project found no difference in survival between clinical stages N0, N1 and N2 [206]. Clinical staging underestimated $\mathrm{N}$ status, subsequently found at surgery, in $33 \%$ of cases and overestimated it in 6\%. Nodal size and the likelihood of malignant involvement have not been found to be correlated [207]. Nodal stage may be predicted from tumour volume. Patients with tumour maximal thickness of $<5.1 \mathrm{~mm}$ had a $14 \%$ risk of nodal metastases, whereas this risk rose to $38 \%$ in patients with tumours of maximal thickness $>5.1 \mathrm{~mm}(\mathrm{p}<0.0001)$ [204].

Invasive mediastinal nodal staging with endobronchial ultrasound (EBUS) or mediastinoscopy can aid clinical staging, but clinicians should be aware that it may not be possible to access all nodal disease, extramediastinal areas (i.e. internal mammary), peridiaphragmatic or intercostal areas.

Task force experts consider that the use of noninvasive imaging is inaccurate in the assessment of nodal metastasis, and even direct biopsy may not exclude occult nodal disease. Therefore, clinicians should be aware of the implications of these staging limitations when discussing pretreatment prognosis.

M stage

The IASLC project evaluated only $84 \mathrm{cM}$ cases, which nevertheless had sufficiently poorer prognosis than cT4 cases to be considered as the only descriptor in the stage IV classification. Exploratory analyses suggested a possible difference in survival for single- versus multiple-site cM1 cases [205].

Task force experts consider that it is important to exclude occult distant metastases if radical therapy is considered due to poor prognosis associated with stage IV.

\section{Pathological staging}

T stage

There appear to be no survival differences between pT1, pT2 and pT3, but there was between pT3 and pT4 (hazard ratio $(\mathrm{HR}) 1.34, \mathrm{p}<0.0005)$ [204]. The classification of pT3 was most often due to partial-thickness pericardial invasion, and pT4 was most commonly due to diffuse chest wall involvement. Other variables that may have prognostic significance include tumour involvement of previous biopsy or incision sites $[186,208]$ and the weight of tumour resected [209].

Clearly marked anatomical structures (pericardium, chest wall biopsy sites) on resection allow accurate pathological orientation and staging, particularly in lung-sparing operations. Any previous biopsy site should always be excised and submitted for histology.

\section{N stage}

The pattern of lymphatic drainage of the pleura does not follow the same pathway as for the lung parenchyma; mediastinal nodes may be the initial site of metastases before the lung parenchyma is involved. Traditional pN2 may therefore precede $\mathrm{pN} 1$.

The IASLC staging project reported no survival difference between pN1 and pN2. Therefore, clinical and pathological N1 and $\mathrm{N} 2$ are combined into a single N1 category including all ipsilateral, intrathoracic nodal metastases. Contralateral or all extrathoracic nodal metastases are then categorised as N2 [206].

The importance of extramediastinal nodal metastases in the intercostal and peridiaphragmatic groups remains unknown due to paucity of data. The proportion of involved versus normal lymph nodes has been found to be more prognostic than anatomical location [210]. 
Pretreatment staging investigations

The stage of the disease determines whether the direction of intervention is cancer-directed (in order to prolong cancer-specific survival) or merely palliation of symptoms. This decision of how extensive the staging measures are will be determined by an initial assessment of the patient's fitness for either surgery or (chemo)therapy. Other factors include the underlying cell type of the tumours (epithelioid versus non-epithelioid) and the TNM staging.

\section{Noninvasive staging}

A summary of noninvasive staging is presented in figure 1.

Semiautomated tumour volume calculations on chest CT scan have correlated volume with pTN stages and overall survival [211].

Fludeoxyglucose (FDG)-PET is limited in the assessment of nodal stage due to the close proximity of diseased pleura, masking uptake. Moreover, previous chemical pleurodesis might affect FDG uptake and maximum standard uptake value $\left(\mathrm{SUV}_{\max }\right)$ measurement. However, it may be useful in the identification of occult distant metastatic disease. PET-CT had low sensitivity for stage N1 (38\%) and T4 (67\%) disease [177]. PET-CT had a higher specificity for stage II (77\% versus $100 \%$, p $<0.01)$ and stage III $(75 \%$ versus $100 \%, \mathrm{p}<0.01$ ) disease compared to $\mathrm{CT}$ alone [212]. SUV $\max$ may be of prognostic significance, even in unresectable disease [213].

MRI may be useful at the margins of the disease: the apex around the subclavian vessels, inferiorly around the diaphragm in order to demonstrate unresectable, multifocal chest wall invasion [177]. Although MRI is superior for detection of brain metastases and bone invasion, this technique was not superior to CT in terms of detection of lymph node metastases $(p=0.85)$ and visceral pleural tumour $(p=0.64)$. PET-MRI may be at least as accurate as PET-CT in staging [214], whereby radiologists felt significantly more confident staging PET-MRI compared to PET-CT using dedicated sequences. Further applications of functional MRI remain research areas only at present [215].

Invasive staging

A concurrent mediastinal nodal biopsy technique by mediastinoscopy has been described [216].

While extramediastinal nodes are anatomically inaccessible, there may be some benefit in excluding those with positive upper mediastinal nodes, as they carried a worse prognosis than lower or extramediastinal areas [208]

EBUS has been found to have superior sensitivity and negative predictive value to mediastinoscopy for nodal disease in MPM. However, values were both $<60 \%$ for EBUS [217]. The theoretical additional yield

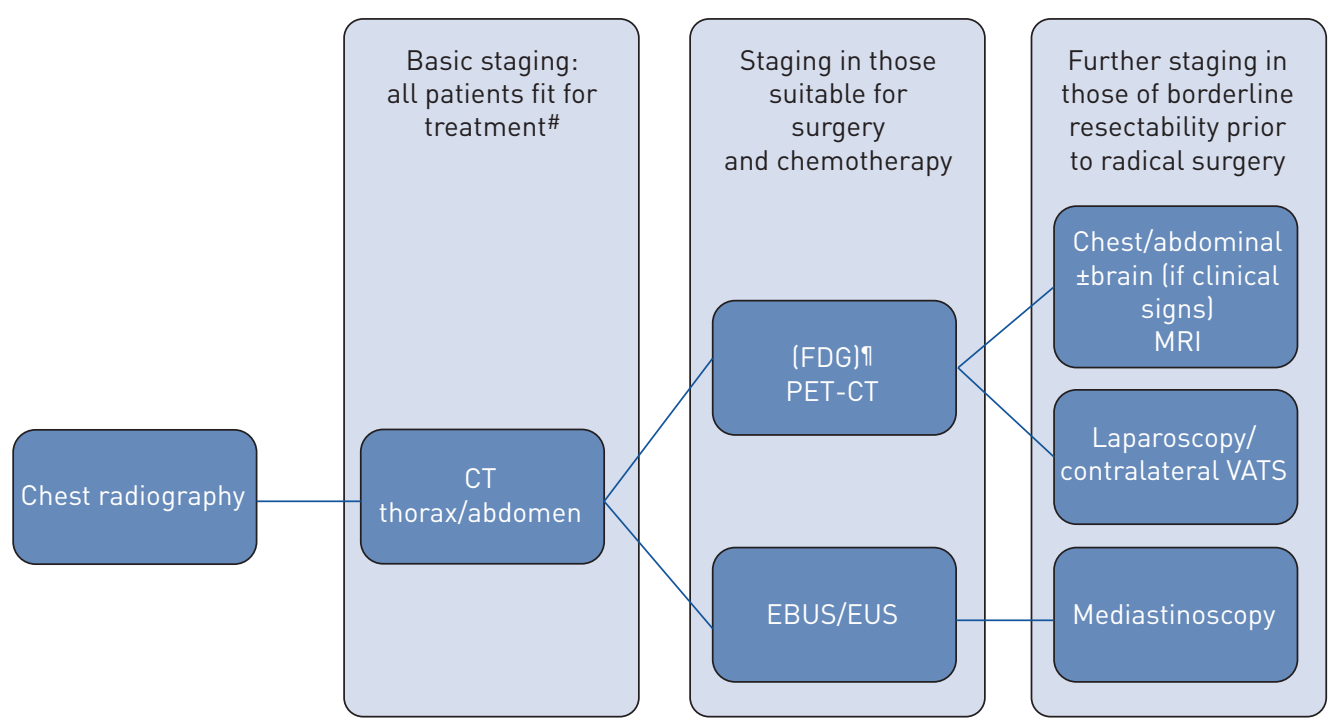

FIGURE 1 A summary of staging algorithm for patients with malignant pleural mesothelioma. * : patients unfit for any treatment could derive some benefit from basic computed tomography (CT) scan in terms of palliative therapy (pleurodesis) or reparation; " : after talcage, positron emission tomography (PET)-CT is less accurate than functional magnetic resonance imaging (MRI). FDG: fludeoxyglucose; EBUS: endobronchial ultrasound; EUS: endoscopic ultrasound; VATS: video-assisted thoracic surgery. 
from EBUS in stations not accessible to mediastinoscopy was $26 \%$, with a mean survival not significantly worse than those within range of mediastinoscopy. Those with only extramediastinal lymph node metastases had a significantly better survival than either of the above groups [218].

EBUS/endoscopic ultrasound (EUS) followed by simultaneous transcervical extended mediastinal lymphadenectomy and laparoscopy/peritoneal lavage revealed only a small number of undetected nodal metastases that were not found by EBUS/EUS, and the majority of those with positive laparoscopy also had positive mediastinal nodes. This algorithm did not include PET-CT [219].

More invasive techniques including contralateral thoracoscopy and laparoscopy have been infrequently used and are difficult to appraise [220]. They have been shown to help identifying occult stage IV disease not seen on PET-CT.

The task force experts consider that the algorithm proposed in figure 1 is a reasonable approach for pretreatment staging investigations. However, it is not intended as a recommendation for clinical practice.

Research priority: the prospective use of volumetric assessment software should be encouraged.

\section{Which other prognostic factors are of importance?}

There is consistent evidence that cell type of MPM is of prognostic significance with epithelioid tumours offering superior survival to non-epithelioid subtypes.

Several nonanatomical prognostic variables can be used to influence the selection of treatment including chest pain, weight loss and dyspnoea, leading to poor performance status, anaemia, leukocytosis and thrombocytosis [221]. Composite prognostic scoring indices have been derived by several organisations including the European Organisation for Research and Treatment of Cancer (EORTC) [222] and Cancer and Leukemia Group B (CALGB) [223] to categorise patients and guide treatment decisions. Specific prognostic scores for surgically resected disease have also been calculated using similar variables: tumour volume pre-(chemo)therapy, C-reactive protein (CRP) level, nonepithelioid histology and progressive disease according to modified Response Evaluation Criteria in Solid Tumours (RECIST) criteria after induction (chemo)therapy [224].

Another simple, clinically relevant model, called the Brims score [225], was proposed to evaluate patients' prognosis using routinely available parameters at the time of diagnosis. This model defined four risk groups with significant different outcomes $(\mathrm{p}<0.0001)$. The strongest predictive variable was the presence of weight loss. Risk group 1 included the patients with the best survival at 18 months $(86.7 \%$ alive, median overall survival (overall survival) of 34.0 months); these patients had no weight loss, a haemoglobin level $>153 \mathrm{~g} \cdot \mathrm{L}^{-1}$, and a serum albumin level $>43 \mathrm{~g} \cdot \mathrm{L}^{-1}$. Risk group $4 \mathrm{~d}$ had the worst outcome ( $0 \%$ alive, median survival 7.5 months); these patients had weight loss, a performance score 0 or 1 , and sarcomatoid histological MPM subtype.

Finally, the PROMISE score was proposed recently as a prognostic score in cohorts of patients with malignant pleural effusion in which a number of patients had mesothelioma [226].

The task force experts consider that prognostic factors and scoring systems may help in the decision process, but cannot usually be applied per se on an individual basis outside clinical trials, as they were not validated for this purpose.

Research priority: the routine use of the Brims score is encouraged, and combined with other scores as part of clinical trials for prospective validation.

In the future, patient-reported outcome measures may potentially improve the management of MPM based on a recent literature survey [227]. There is also a need to derive predictive factors of (chemo)therapy.

\section{Treatment of MPM}

Surgery for MPM patients

Should partial pleurectomy compared to talc pleurodesis be used as palliative procedure in patients with symptomatic MPM?

Our systematic review identified one randomised controlled trial (MesoVATS trial) [228] that compared partial pleurectomy (PP) by VATS versus talc pleurodesis in patients with MPM. The MesoVATS trial was an open-label randomised controlled trial conducted in 12 centres in the UK. The primary outcome was overall survival at 1 year. There were no differences between groups in the overall survival at 1 year (HR 1.04, 95\% CI 0.76-1.42) nor at 6 months follow-up. Surgical complications were significantly more common after VATS-PP than after talc pleurodesis, occurring in $24(31 \%)$ out of 78 patients who completed VATS-PP versus 10 (14\%) out of 73 patients who completed talc pleurodesis ( $\mathrm{p}=0.019)$. Median (interquartile range) hospital stay was longer at 7 (5-11) days in patients who received VATS-PP compared with 3 (2-5) days for 
those who received talc pleurodesis $(\mathrm{p}<0.0001)$. However, the proportion of patients with resolved pleural effusion was significantly higher in the PP group than in the talc pleurodesis group at 1 month (37\% versus $59 \%$ ), but not at 3 months (60\% versus $60 \%$ ) or 12 months (77\% versus $70 \%$ ), although these numbers were based on surviving patients and heavily influenced by the attrition of follow-up (supplementary table S14). Furthermore, the benefits of VATS-PP (better quality of life, less short-term pleural effusion) do not balance the inconveniences (more leaks and cost). These data do not support a change of practice.

Recommendation: we recommend talc poudrage via thoracoscopy to control a recurrent MPM effusion as the first choice to achieve pleurodesis in patients with expanded lungs (strong recommendation, low quality of evidence).

We suggest palliative VATS-PP to obtain pleural effusion control in symptomatic patients fit enough to undergo surgery who cannot benefit from (or after failure of) chemical pleurodesis or indwelling catheter (weak recommendation, low quality of evidence).

Should radical surgery (including extrapleural pneumonectomy or pneumonectomy/decortication) be used in patients with MPM?

Radical surgery in MPM is defined as macroscopic complete resection, which can be achieved by extrapleural pneumonectomy (EPP) consisting of en bloc resection of pleura, lung, pericardium and diaphragm combined with systematic mediastinal lymph node dissection, or (extended) pleurectomy/ decortication (P/D) and systematic mediastinal lymph node dissection. P/D is a resection of the total parietal and visceral pleurectomy, sparing the pericardium and the hemidiaphragm, while extended pleurectomy/decortication (EP/D) includes the resection of the pericardium and the hemidiaphragm, when required, and in order to remove all the macroscopic disease [229].

Whereas population and cancer registries consistently report a better outcome for surgically treated patients, they do not correct for prognostic factors, or do so incompletely, and are hence subject to patient selection and recall bias [230-235].

Our systematic review identified one randomised controlled trial (Mesothelioma and Radical Surgery (MARS) trial) [236] and two observational studies [237, 238] that compared surgical to nonsurgical therapeutic approaches in patients with MPM. The MARS trial was designed as a feasibility study and underpowered to assess any benefit (or absence thereof) of EPP. The low number of patients and the number of registered events was very limited; these features decreased the panel's confidence in the estimated effects to low. The study showed that the adjusted HR for overall survival between the EPP and no-EPP groups was 2.75 (95\% CI 1.21-6.26). At a median follow-up of 24.7 months from randomisation, 30 out of 50 patients had died (EPP $n=17$; no EPP $n=13$ ); thus, the analysis of survival included only 30 deaths. The 12-month recurrence-free survival in the EPP group was 34.8\% (95\% CI 16.6-53.7\%) compared to $42.3 \%$ (95\% CI $23.5-60.0 \%$ ) in the no EPP group, although the difference was not statistically significant. There were no statistically significant differences in those patients who completed the quality-of-life assessment (EPP $n=12$; no EPP $n=19$ ), although the median quality-of-life scores seemed to be lower for the EPP group than the no-EPP group. 12 serious adverse events were reported during the study period: 10 in the EPP group and two in the no-EPP group. Further critical problems are that the total number of patients achieving the trimodality approach was very low, and a relevant number of no-EPP patients received EPP (supplementary table S15).

These results differ from a large retrospective cohort of 1365 consecutive patients with MPM, suggesting that patients with good prognostic factors (i.e. age $<70$ years, epithelioid histology) have similar survival, whether they receive medical therapy only, P/D or EPP [237] (supplementary table S16).

Another retrospective study in 150 patients showed a nonsignificant trend to better overall survival and disease-free survival in those patients undergoing surgical resection (P/D or EPP) [238].

One bias of retrospective studies is that the choice of P/D or EPP depends largely on the institutions' experience, because of a huge variability of outcomes reporting regarding morbidity, mortality, quality of life and overall and disease-free survival. Therefore, due to the low overall confidence and the conflicting results between studies, the panel did not consider issuing a recommendation until more consistent data become available. A multicentre randomised trial comparing extended P/D to no surgery (MARS-2 trial) is currently recruiting in the UK [239]. Results from this surgical trial are awaited with interest.

Research priority: patients considered for radical surgery should be either included in prospective randomised controlled clinical trials or in national/international surgical registries.

Remark: surgery may be appropriate for carefully and highly selected MPM patients. This would usually be EP/D rather than EPP, because of its lower comparative respiratory postoperative morbidity and 
preservation of quality of life, performed in centres of excellence and as part of multimodality treatment. Patients with sarcomatoid or sarcomatoid-predominant histology, N2 disease (8th edition TNM staging system) and/or stage IV should not be considered for radical surgery other than in the context of research. However, as no single prognostic factor influences treatment allocation, prognostic scores encompassing several prognostic factors should be preferred (see sections on staging and allocation).

\section{Radiotherapy of MPM}

Should radiotherapy be used for pain relief in patients with MPM?

Evidence from randomised controlled trials is not available for palliative radiotherapy in MPM. A prospective multicentre single-arm study [240] investigating $20 \mathrm{~Gy}$ in five fractions to painful areas in 40 patients demonstrated that radiotherapy can be effective in treating pain in selected mesothelioma patients (number needed to treat=2). Despite very limited data in the setting of MPM, the role of radiotherapy in pain control for other solid tumours has been demonstrated and is accepted in clinical routine [241-243].

Recommendation: we suggest that palliative radiotherapy for pain relief should be considered in cases of painful sites of disease caused by local infiltration of normal structures (moderate recommendation, low quality of evidence).

\section{Should radiotherapy be used to prevent procedure-tract metastases (drain site parietal seeding) in} patients with MPM?

Randomised controlled trials investigating prophylactic drain site radiotherapy in MPM have shown contradictory results. BouTin et al. [244] previously showed that an irradiation with $21 \mathrm{~Gy}$ in three fractions for three consecutive days in the 4 weeks following drainage or thoracoscopy prevents subcutaneous metastasis developing along drainage channels or thoracentesis tracts. However, a subsequent randomised trial was published comparing immediate drain site radiotherapy 21 Gy in three fractions to no radiotherapy in 61 patients treated between 1998 and 2004, with no difference in terms of tract metastatic recurrence between the two arms [245, 246]. O'RouRKe et al. [245] concluded that prophylactic drain site radiotherapy in MPM did not reduce the incidence of tumour seeding as indicated in previous studies [247, 248].

Since the last guideline, two further randomised studies were not able to demonstrate a benefit with prophylactic tract irradiation. A multicentre phase III trial [249] compared immediate radiotherapy (21 Gy in three fractions within 42 days of the pleural intervention) with deferred radiotherapy (same dose given within 35 days of diagnosis of procedure-tract metastases (PTM)); 203 patients were randomised. There was no significant difference in terms of PTM rate, chest pain, quality of life, analgesia requirements or survival. However, there was a suggestion of a benefit in two predefined subgroup analyses, i.e. patients with epithelioid-only histology and those who did not receive (chemo)therapy (supplementary table S17).

The applicability of these findings is limited by the small numbers, thus further studies in these specific subgroups may be warranted. A further multicentre phase III randomised trial randomised 375 patients to prophylactic irradiation of tracts $(21 \mathrm{~Gy}$ in three fractions within 42 days of the pleural intervention) or not. At 12 months, the rate of tract recurrence was $8.1 \%$ versus $10.1 \%$, respectively ( $p=0.59)$ [250]. Prophylactic radiotherapy did not have a statistically significant reduction on the risk of procedure site recurrence, with a pooled relative risk of 0.64 (95\% CI $0.27-1.51$ ).

While the results of these two large randomised controlled trials can be considered contradictory to older and smaller trials of the pre(chemo)therapy era, the limited effects of radiotherapy to the prophylactic drain sites observed in these UK phase III trials do not justify this procedure in routine practice.

Recommendation: we do not recommend prophylactic drain site radiotherapy in routine clinical care (strong recommendation, moderate quality of evidence).

\section{Should adjuvant postoperative radiotherapy be used in patients with MPM?}

The 17/04 SAKK trial (Neo-adjuvant Chemotherapy and Extrapleural Pneumonectomy of MPM With or Without Hemithoracic Radiotherapy) randomised 54 patients post-EPP to observation versus adjuvant (minimum dose of $50 \mathrm{~Gy}$ with daily fraction size of 1.8-2 Gy) [251]. The trial closed earlier than planned due to poor accrual. Radiotherapy was associated with slightly better median locoregional relapse-free survival (9.4 months versus 7.6 months); however, this was not statistically significant (supplementary table S18).

A phase I/II trial has demonstrated that a short accelerated course of high-dose hemithoracic intensitymodulated radiation therapy (IMRT) followed by EPP is feasible [252]. Patients received 25 Gy in five daily fractions over 1 week to the entire ipsilateral hemithorax with concomitant 5 Gy boost to areas at risk followed by EPP within 1 week of completing neoadjuvant IMRT. Patients with epithelioid histological subtypes had a 
3-year survival of $84 \%$ after a median follow-up of 23 months. While these results are encouraging and warrant further investigation, this approach is considered experimental at this point. Radiation therapy after lung-sparing surgery might be another approach, resulting in promising survival data [253].

A phase II study [254] demonstrated that hemithoracic pleural IMRT for MPM is safe and has an acceptable rate of side-effects. Its incorporation with (chemo)therapy and P/D forms a new lung-sparing treatment paradigm for patients with locally advanced MPM, but randomised trials are needed to potentially establish this in clinical routine.

Research priority: radiotherapy after pleurectomy \pm decortication or after EPP should only be considered within the context of clinical trials and/or included in national/international surgical registries.

\section{Medical treatment of MPM}

Some phase II and III trials have been completed in first-line and salvage therapy since the 2009 ERS/ESTS guidelines [255]. They are presented in supplementary tables S12 [256-274] and S13 [256, 259, 260, 275-290].

\section{Should first-line (chemo)therapy consisting of platinum in combination with pemetrexed be used in} patients with MPM?

No innovative drug has been validated in MPM since 2009 [255].

Recommendations (unchanged after the previous guidelines [8]): we recommend first-line combination (chemo)therapy consisting of platinum and pemetrexed (with folic acid and vitamin B12 supplementation) in patients fit for (chemo)therapy (good performance status, ECOG performance status 0-2, no contraindications) (strong recommendation, low quality of evidence).

Remarks: the administration of (chemo)therapy should not be delayed and should be considered before the appearance of functional clinical signs (or clinical deterioration). Chemotherapy should be stopped in the event of progressive disease, grade 3-4 toxicities or cumulative toxic doses, but should be continued up to six cycles in patients who respond or are stable.

Research priority: patients demonstrating prolonged symptomatic and objective response with first-line pemetrexed-based (chemo)therapy may be treated again with the same regimen in the event of recurrence. In the remainder of cases, inclusion of the patients in clinical trials is highly encouraged.

\section{Should bevacizumab or other targeted therapies be added to first-line standard (chemo)therapy in patients with MPM?}

In 2009, the guidelines task force concluded that immunomodulating agents, targeted therapies and vaccines should not be used in the treatment of MPM outside clinical trials. Many targeted therapies have been assessed in MPM since this time (reviewed in $[2,3]$ ), including mainly antiangiogenic drugs and other growth factor inhibitors.

A large ( $\mathrm{n}=448)$, phase III trial (Mesothelioma Avastin Cisplatin Pemetrexed Study (MAPS)) showed benefit in adding bevacizumab to cisplatin (cis)/pemetrexed (pem) doublet as first-line treatment [271] with significantly longer survival (primary end-point) (HR 0.67, 95\% CI 0.61-0.94; $\mathrm{p}=0.015$ ) and a 2-month increase in progression-free survival (PFS) (HR 0.61, 95\% CI 0.50-0.75; $<<0.0001$ ) favouring the bevacizumab arm, with only a mild and manageable increase of toxicity and no negative impact on quality of life. This study suggested a new standard of care for unresectable MPM patients, as validated by some US (NCCN) and French guidelines. However, to date, bevacizumab has not received US Food and Drug Administration or European Medicines Agency approvals in MPM because the French Cooperative Thoracic Intergroup MAPS trial was an academic trial, not initially designed for registration purposes (supplementary table S20).

No other antiangiogenic drug or tyrosine kinase inhibitors has yet demonstrated significant efficacy in a randomised phase III trial [3]. Thus, nintedanib, a drug targeting vascular endothelial growth factor receptor $1-3$, platelet-derived growth factor receptor- $\alpha /-\beta$ and fibroblast growth factor receptor $1-3$ failed to show any value in the phase III LUME-Meso trial [291] despite previous promising results in a randomised phase II trial versus placebo in conjunction with first-line cis/pem [292] with significant improvement in median PFS (HR 0.54) and in median overall survival (HR 0.77) (supplementary table S21).

Other main targeted drugs evaluated in MPM included vorinostat, an inhibitor of histone deacetylases, which failed to show any survival advantage versus placebo as second- or third-line treatment in a large phase III trial [284]. The phase II COMMAND trial (NCT01870609), assessing the focal adhesion kinase inhibitor VS-6063/defactinib versus placebo as maintenance treatment after first-line cis/pem, did not meet its primary goals (median PFS and median overall survival) [293]. Other promising drugs include pegylated arginine deaminase (ADI-PEG 20), in combination with cis/pem, targeting arginosuccinate 
synthetase-1-deficient tumours such as biphasic (mixed) or sarcomatoid MPM [294]; the loss of BAP-1 may induce the sensitivity of MPM cells to therapies targeting the EZH2 pathway.

Recommendation: we suggest that bevacizumab, if available, be proposed in combination with cisplatin/ pemetrexed as first-line treatment in patients fit for bevazucimab and cisplatin, but not for macroscopic complete resection (weak recommendation, moderate quality of evidence).

Should immunotherapy be used as salvage therapy in patients with MPM who failed first-line standard (chemo)therapy?

Since 2009, new immunotherapies have been tested in MPM, in particular immune checkpoint inhibitors such as anti-CTLA-4 (ipilimumab, tremelimumab), anti-PD-1 (pembrolizumab, nivolumab) and anti-PD-L1 (durvalumab, avelumab). Tremelimumab failed to show any survival improvement versus placebo as second-line treatment in a phase III trial [289] (supplementary table S22). In preliminary data from small nonrandomised trials, anti-PD-1 or anti-PD-L1 antibodies seemed to induce increased overall response rate and overall survival compared to historical second- or third-line chemotherapies [3, 295]. PROMISE MESO (NCT02991482), a phase III trial comparing pembrolizumab versus either vinorelbine or gemcitabine, has completed enrolment. CONFIRM (NCT03063450), a phase III double-blind randomised trial evaluating nivolumab versus placebo is ongoing $[3,296]$. Moreover, in the same setting, nivolumab alone or combination of nivolumab plus ipilimumab significantly increased the disease control rate after 12 weeks of treatment and overall survival in a randomised phase II trial [297]. This combination was also efficient in another mono-arm phase II trial as second- or third-line treatment for MPM [298]. Durvalumab and tremelimumab combination may also have a therapeutic value in MPM patients, based on a first report [299]. Finally, preliminary reports of first-line (chemo)therapy plus anti-PD-1 or anti-PD-L1 are promising [300].

Several other trials are ongoing [301], assessing immunotherapies, alone or combined with (chemo) therapy and/or targeted therapies (anti-angiogenic, epigenetic drugs), as first-line or salvage therapies. Interestingly, cell therapy (with dendritic cells, chimeric antigen receptor (CAR) T-cells) or gene therapy trials are also currently recruiting MPM patients.

Research priority: novel insights in immunotherapy are promising, but need further development and results from ongoing or planned phase III trials before any definitive recommendations can be made for their use in the clinical routine. Inclusion of patients in these trials is highly recommended.

What assessment criteria should be used to determine the efficacy of systemic treatment in MPM?

No specific significant data have been published since the previous guidelines [255]. The activity of a treatment can be assessed on clinical criteria (symptoms control and quality of life), imaging criteria (CT scan, PET scan) and survival criteria (time to progression, overall survival).

Overall survival is not the only valuable parameter to assess the effectiveness of medical treatment in clinical trials. It is recommended that quality of life and symptom control be taken into account, to evaluate the clinical benefit (efficacy/tolerance) in diseases with poor prognosis and for which the survival impact of the treatment is not clearly demonstrated or is marginal. No particular score to assess quality of life is recommended specifically, except the modified version of the Lung Cancer Symptom Scale adapted to patients presenting with malignant mesothelioma.

For clinicians MPM is characterised by obstacles in tumour measurement and response assessment. To help them in routine practice as well as in the conduct, interpretation and reporting of clinical trials, the modified RECIST was proposed in 2004. However, the practical application of these criteria was tricky, leading to misinterpretation and inconsistencies in tumour response assessment. Therefore, the modified RECIST 1.1 for mesothelioma [302] were proposed recently to provide updated response assessment guidelines improving previous criteria but also aiming at better defining crucial concepts for MPM, such as minimally measurable disease, measurable lesions, acceptable measurement location or nonmeasurable pleural disease. In addition, they may help to better evaluate nonpleural disease, pathological lymph nodes and bilateral MPM and to establish progressive disease.

Even if they have not been prospectively validated, the task force experts consider the updated modified RECIST 1.1 guidelines the preferred method of choice for measuring tumour lesions and response to treatment on CT scans. If a patient has had pleurodesis, it has been strongly suggested that a chest CT scan should be repeated before the start of (chemo)therapy in order to better evaluate the response to treatment. In fact, pleural lesions may be better described after removal of pleural effusion, favouring a correct assessment of patient outcome. PET scan and biological markers are still under investigation for the evaluation of treatment response in MPM. 
Should a multimodal therapy approach (combining more than one method of cancer treatment: surgery, (chemo)therapy, radiation therapy) compared to (chemo)therapy alone be used in patients with MPM?

In order to address the role of multimodality therapy in MPM, the following clinical questions were raised. Is multimodality treatment better than (chemo)therapy alone? What is the optimal regimen within each modality? What is the optimal sequence of interventions within a combined modality approach? However, since 2009, our systematic review of the literature, as well as two other recent reviews [303, 304] only identified two randomised clinical trials on the topic: MARS and SAKK 17/04 [236, 251]. Both trials have been considered in other sections of these guidelines (radical surgery and postoperative radiotherapy), without mentioning that they were assessing multimodality options, leading the task force to only issue research priorities. These two trials had many weaknesses. For example, the MARS study was a feasibility trial that did not reach the prespecified sample size [236]; multimodality treatment was compared to continued oncological management, which could include (chemo)therapy and palliative radiotherapy [236], or (chemo)therapy and surgery [251]. Median overall survival observed in both studies was less than expected when compared with observational data; this result might partly be explained by the inclusion of patients with worse prognosis. Globally, these trials involved limited number of patients and events, and wide $95 \%$ confidence intervals that included appreciable harm or benefit (supplementary tables S15 and S18).

Thus, as emphasised by other recent reviews [303, 304] or guidelines [4-6], the literature remains biased for multimodal management of MPM patients, without high quality of evidence in favour of a specific therapeutic combination or scheme. Multimodal treatment consisting of at least macroscopic complete resection and (chemo)therapy (platinum/pemetrexed doublet), was superior to either single modality in selected patients with regard to survival, but at the cost of increased treatment-related morbidity and mortality [304]. Given the added cost of multimodality strategies, the possible increase in risk of adverse effects and the lack of evidence of their effectiveness, the Cochrane review authors also concluded that these interventions should not be proposed in routine clinical practice.

Research priority: we still recommend that patients who are considered candidates for a multimodal approach should be adequately informed of its challenges and referred to expert centres in order to be included in a prospective (randomised) clinical trial and/or registered in a large institutional database.

\section{Treatment allocation}

This question, as well as the global management of MPM patients, is summarised by the algorithm presented in figure 2. Counselling patients for the most appropriate and promising treatment, balancing life expectancy with quality of life remains a difficult issue, despite the development of a more detailed TNM staging [200, 305, 306], progress in staging tools and improved knowledge of tumour biology. In contrast to most other malignancies, the discrepancy in reliability between clinical and pathological staging leads quite frequently to an unsatisfactory patient selection for multimodality treatment including radical surgery. When radical surgery (usually P/D) is considered, clinical and functional assessment should be undertaken as described above, including at least spirometry, diffusion capacity of the lung for carbon monoxide, and cardiovascular assessment. CT, PET-CT and/or MRI are used to exclude distant metastasis and evaluate resectability. Thus, the decision whether radical surgery is recommended should be based on a number of different aspects. It has been shown in various studies [211] that tumour volume, measured preoperatively on CT scans, predicts $\mathrm{pT} / \mathrm{pN}$ and overall survival. Other single factors such as mediastinal nodal involvement or histology available preoperatively (see staging section) predict overall survival. Despite an increasing knowledge about molecular markers and their diagnostic and prognostic value, they are not yet used for treatment allocation. Not surprisingly, single factors are insufficient for proper treatment allocation, and prognostic scores have been developed. The EORTC and the CALGB [222, 223] scores were developed for better identification of patients receiving (chemo)therapy. Prognostically relevant "CORE" covariates (stage, sex, age, histology and type of surgery) were evaluated for patient selection [221]. A multimodality prognostic score based on tumour volume, histology, CRP at diagnosis, nodal status and response to (chemo)therapy allows the identification of patients with very poor prognosis despite multimodality therapy [224]. In conclusion, several prognostic scores have been proposed for treatment allocation of MPM patients. But to date, no single parameter or score has been widely validated for routine use for this purpose.

Research priority: current and future scores suggested for patient treatment allocation, always decided by an MPM expert multidisciplinary board, require prospective validation by multicentre studies.

\section{Palliative care}

The control of malignant pleural effusion (MPE) is not detailed in these guidelines, as it is fully explained in the new ERS/EACTS guidelines on MPE management [307]. 


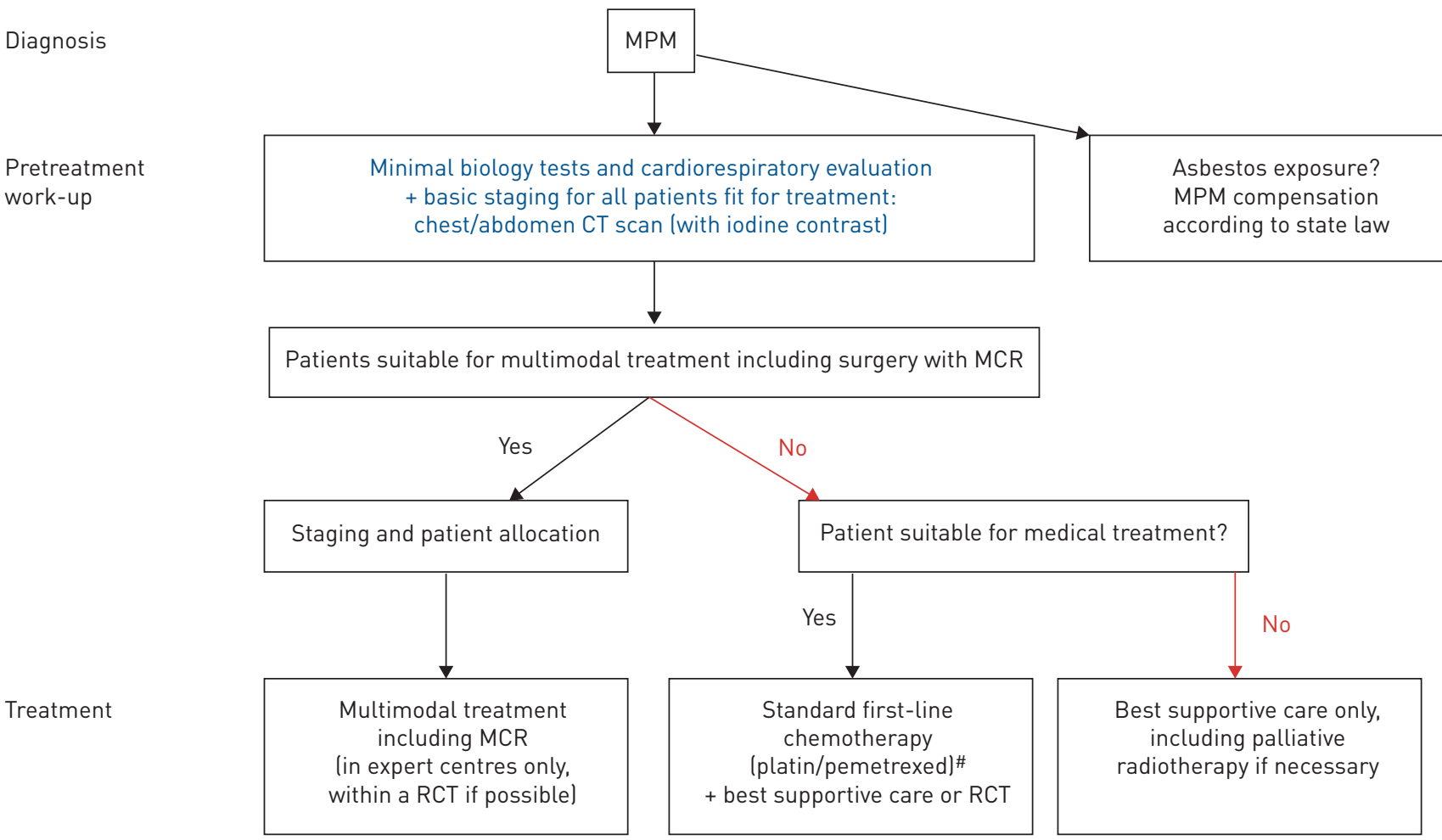

FIGURE 2 A simplified algorithm for the management of patients with malignant pleural mesothelioma (MPM). RCT: randomised controlled trial; MCR: macroscopic complete resection. ${ }^{\#}$ : \pm bevacizumab if available and no contraindication.

Good-quality palliative care is vital for MPM patients, the majority of whom will require symptom control at some stage in the course of their disease. Currently there are no published large randomised controlled studies of symptom control in patients with MPM only. A small prospective randomised (1:1) phase II trial assessed the use of early versus delayed (chemo)therapy at time of symptomatic progression after best supportive care (BSC) only in 43 patients, presenting with stable symptoms after control of pleural effusion [308]. The early use of (chemo)therapy provided an extended median time to symptomatic progression versus the delayed (chemo)therapy group ( 25 versus 11 weeks, $\mathrm{p}=0.1$ ), and a trend to survival advantage (median overall survival of 14 months versus 10 months, $\mathrm{p}=0.1$ ).

There are a two relatively unique problems experienced by a proportion of mesothelioma patients. 1) Excessive sweating: no RCT studies have been published in this field, but it remains a common problem in a proportion of mesothelioma patients. Although there are no good-quality data, oral prednisolone can be very effective in helping to reduce this disabling symptom; 2) severe unilateral thoracic pain: a case series of 53 patients with MPM and associated persistent pain despite oral analgesia were managed with cervical cordotomy [309]. The majority of patients had a reduction in pain following the procedure; however, further, more robust studies are required to confirm this finding.

A review of the numerous palliative care intervention for patients with MPM was out of the scope of this guideline. Therefore, the task force experts encourage following existing national palliative care guidelines for guidance on pain control in cancer patients.

The task force experts emphasise that it is recognised that mesothelioma is associated with high psychological burden, and although quantitative evidence is sparse, there are qualitative papers and systematic reviews that demonstrate this [310].

\section{Follow-up after active treatment}

There are no evidence-based recommendations regarding the follow-up in mesothelioma patients undergoing a dedicated treatment mainly based on (chemo)therapy. Although (chemo)therapy has been shown to benefit patients, there are no consistent data allowing us to answer the question of the optimal duration of (chemo)therapy and the design of patients' survey after cessation of the treatment. Therefore, symptoms such as breathlessness, chest pain or both indicate re-evaluation by CT scan to search for progressive disease $[308,311,312]$. Other main symptoms consist of cough (frequently due to pleural effusion), anorexia, weight loss, fatigue, sweating, dysphagia and psychological distress. There are no data 
showing the place of PET and MRI in the follow-up for MPM. The development of targeted therapies and immunotherapy in a near future would probably lead clinicians to adapt the modalities of follow-up for mesothelioma patients [313]. To date, there is no sufficient evidence for routine use of biomarkers such as blood mesothelin or other markers for follow-up of MPM patients, either to predict the response to treatment or patient outcomes.

Research priority: the role of periodic follow-up with imaging (chest/abdominal CT scan, MRI or PET) should be assessed in clinical trials.

Remarks: monitoring of disease progression should be guided by signs and symptoms occurring during clinical follow-up. However, in addition to clinical follow-up, and pending further evidence from clinical trials, the task force group suggests a chest/abdominal CT scan every 3-6 months after active treatment of MPM patients.

\section{The outlook for MPM}

After a decade during which systemic therapy for mesothelioma has languished at a therapeutic plateau [314], recent advances have demonstrated that improvement in efficacy can be associated with the addition of novel agents in the context of randomised phase III trials, e.g. bevacizumab [271], but not nintedanib with a negative phase III trial (NCT01907100) despite positive randomised phase II trial results [292]. The role of aggressive local control in the form of extended pleurectomy/decortication will become clearer in the next few years, but positive result of current trials may promote further discussion regarding the radicality of a surgical approach.

Despite these recent advances and awaited results from ongoing surgical clinical trials such as MARS2 (NCT02040272), a major challenge remains in the relapsed setting, where there is currently no approved standard.

Accordingly, translational and clinical research in this setting has the potential to significantly improve survival outcomes. Despite the failure of CTLA-4 checkpoint targeted immunotherapy in relapsed mesothelioma [289], the emerging signals of activity for anti-PD-1 monotherapy [295] and combination PD-1 (or PD-L1)/CTLA-4 targeted therapy [297], indicate some potential for these approaches in the relapsed and potentially frontline settings [296], as demonstrated in other cancers such as melanoma [2, 3]. However, the MAPS-2 trial reported a higher incidence of grade 3 or 4 adverse events $(26.2 \%$ versus $12.7 \%$ ), and even three toxic deaths, with the combination nivolumab/ipilimumab versus nivolumab alone, respectively [315]. This toxicity issue and the choice of inadequate surrogate end-points such as PFS instead of overall survival must be taken into account when assessing the value of new drugs in MPM [316].

Thus, a major challenge for the field as a whole, will be how best to predict the efficacy of both monotherapy and combination immune checkpoint inhibition. This is particularly important from a health economic standpoint to ensure that advances are ultimately affordable, as well as driving up the efficacy of therapy through enrichment of those likely to respond. Meeting this challenge will require assessment of established predictive biomarkers such as PD-L1, but also the role of other potential predictors including tumour infiltrating lymphocytes [317], cytokine expression [318] and tumour mutation burden $[319,320]$, ideally in the context of phase III clinical trials. Exploitation of the abscopal effect could also enhance the efficacy of immunotherapy and warrants exploration [321].

Studies are currently under development in the context of combination with both (chemo)therapy and novel agents [301] (e.g. focal adhesion kinase [322], bevacizumab [323]). Future advances in next-generation combination immunotherapy, e.g. indoleamine 2,3-dioxygenase [324]/T-cell immunoglobulin mucin-3 inhibitors [325]/vaccines, etc. may emerge from the rapid pace of development in basic and translational science and advances in other cancers, as well as tailoring of therapeutic hypotheses based on specific mesothelioma biology, including gene-driven metabolic reprogramming.

Genomic stratification of systemic therapy has revolutionised treatment in other areas including lung and breast cancers. Mesothelioma is lagging behind, partly due to a lack of druggable oncogenic mutations [2]. However, recent advances demonstrate potential opportunities. Arginine auxotrophy, arising from the loss of the citrulline-to-arginine converting enzyme argininosuccinyl synthetase, has recently been shown to be a druggable target $[294,326,327]$ with a phase III trial now enrolling in the front-line setting. Other novel metabolic vulnerabilities may be identified from interrogation of recently available large-scale genomic data that could underpin the development of new synthetic lethal strategies.

Tumour suppressor losses are common in mesothelioma and may have implications for targeted therapy. For example, the discovery that inactivation of the BAP1 tumour suppressor is associated with upregulation of EZH2 [328] or defective homologous DNA repair [329] has led to the development of phase II trials to test this hypothesis. Other preclinical evidence suggests how sensitivity to 
BOX 1 Summary of questions and recommendations

Questions

\section{Epidemiology}

MPM screening

Biomarkers for MPM

\section{Staging}

Clinical staging

Pre-treatment staging investigations

Which other prognostic factors are of importance?

\section{Surgery (PICO)}

Should partial pleurectomy compared to talc pleurodesis be used as a palliative procedure in patients with symptomatic MPM?

Should radical surgery lincluding extrapleural pneumonectomy or pneumonectomy/decortication) be used in patients with MPM?

\section{Radiotherapy (PICO)}

Should radiotherapy be used for pain relief in patients with MPM?

Should radiotherapy be used to prevent procedure-tract metastases (drain site parietal seedingl in patients with MPM?

Should adjuvant post-operative radiotherapy be used in patients with MPM?

\section{Medical treatment (PICO)}

Should first line chemotherapy consisting of platinum in combination with pemetrexed be used in patients with MPM?

Should targeted therapies be added to first line standard chemotherapy in patients with MPM?

Should bevacizumab be added to first line standard chemotherapy in patients with MPM?

\section{Recommendations and research priorities}

Research priority: the relationship between pleural plaques and MPM should be ascertained in large international epidemiological studies. The effectiveness of CT screening in the asbestos-exposed population should be determined in well-designed clinical trials.

Research priority: routine determination of previously proposed biomarkers in MPM have no current validated role in diagnosis, prognosis or clinical follow-up (disease monitoring). Thus, further research into the role of biomarkers in these goals is required and highly encouraged.

Research priority: prospective data collection about the measurement of tumour thickness or volume is to be encouraged.

Research priority: the prospective use of volumetric assessment software should be encouraged.

Research priority: the routine use of the Brims score is encouraged, and combined with other scores as part of clinical trials for prospective validation.

Recommendation: we recommend talc poudrage via thoracoscopy to control a recurrent MPM effusion as the first choice to achieve pleurodesis in patients with expanded lungs (strong recommendation, low quality of evidence).

We suggest palliative VATS-PP to obtain pleural effusion control in symptomatic patients fit enough to undergo surgery who cannot benefit from (or after failure of) chemical pleurodesis or indwelling catheter (weak recommendation, low quality of evidence).

Research priority: patients considered for radical surgery should be either included in prospective randomised controlled clinical trials or in national/international surgical registries.

Remark: surgery may be appropriate for carefully and highly selected MPM patients. This would usually be EP/D rather than EPP, because of its lower comparative respiratory postoperative morbidity and preservation of quality of life, performed in centres of excellence and as part of multimodality treatment. Patients with sarcomatoid or sarcomatoidpredominant histology, N2 disease (8th edition TNM staging system) and/or stage IV should not be considered for radical surgery other than in the context of research. However, as no single prognostic factor influences treatment allocation, prognostic scores encompassing several prognostic factors should be preferred (see sections on staging and allocation).

Recommendation: we suggest that palliative radiotherapy for pain relief should be considered in cases of painful sites of disease caused by local infiltration of normal structures (moderate recommendation, low quality of evidence).

Recommendation: we do not recommend prophylactic drain site radiotherapy in routine clinical care (strong recommendation, moderate quality of evidence).

Research priority: radiotherapy after pleurectomy decortication or after EPP should only be considered within the context of clinical trials and/or included in national/international surgical registries.

We recommend first-line combination (chemo)therapy consisting of platinum and pemetrexed (with folic acid and vitamin B12 supplementation) in patients fit for (chemo)therapy (good performance status, ECOG performance status $0-2$, no contraindications) (strong recommendation, low quality of evidencel.

Research priority: patients demonstrating prolonged symptomatic and objective response with first-line pemetrexed-based (chemo)therapy may be treated again with the same regimen in the event of recurrence. In the remainder of cases, inclusion of the patients in clinical trials is highly encouraged.

Recommendation: we suggest that bevacizumab, if available, be proposed in combination with cisplatin/pemetrexed as first-line treatment in patients fit for bevazucimab and cisplatin, but not for macroscopic complete resection (weak recommendation, moderate quality of evidencel. 


\section{BOX 1 Continued}

Should immunotherapy be used as salvage therapy in patients with MPM who failed first-line standard chemotherapy?

\section{Multimodal treatment (PICO)}

Should a multimodal therapy approach lcombining more than one method of cancer treatment: surgery, chemotherapy, radiation therapyl compared to chemotherapy alone be used in patients with MPM?

\section{Treatment allocation of MPM}

\section{Follow-up of MPM patients}

What should be the follow-up of a patient after active treatment of MPM?
Research priority: novel insights in immunotherapy are promising, but need further development and results from ongoing or planned phase III trials before any definitive recommendations can be made for their use in the clinical routine. Inclusion of patients in these trials is highly recommended.

Research priority: we still recommend that patients who are considered candidates for a multimodal approach should be adequately informed of its challenges and referred to expert centres in order to be included in a prospective (randomised) clinical trial or registered in a large institutional database.

Research priority: current and future scores suggested for patient treatment allocation, always decided by an MPM expert multidisciplinary board, would require prospective validation by multicentre studies.

Research priority: the role of periodic follow-up with imaging (chest/abdominal CT scan, MRI or PET) should be assessed in clinical trials.

Remarks: monitoring of disease progression should be guided by signs and symptoms occurring during clinical follow-up. However, in addition to clinical follow-up, and pending further evidence from clinical trials, the task force group suggests a chest/abdominal CT scan every 3-6 months after active treatment of MPM patients.

chemotherapeutic agents can be BAP1-driven and prompt a future patient stratification to improve the efficacy of standard treatments [330]. Emerging insights into other synthetic lethal interactions with CDKN2A and NF2 have significant translational potential.

Micro-RNAs (MiRs) broadly regulate the transcriptome of mesothelioma and may contribute to the drug-resistant and aggressive phenotype. Recently, MiR16 has been identified as a potential tumour suppressor that can be targeted using so-called targoMiRs. VAN ZANDwiJ et al. [331] reported that MiR-directed targoMiR can be delivered in the clinical setting and can induce responses in relapsed mesothelioma, suggesting that this approach could have therapeutic potential in the future.

The apparently unique treatment-resistant profile of mesothelioma prompts a need for in-depth preclinical research to gain an increased understanding of mesothelioma biology. Potential areas of focus for research include microenvironment-tumour interaction, gene-driven metabolism [329, 332] and elucidation of the mechanisms behind cell death. Preclinical research should use accurate models such as organoids, patient-derived xenografts, primary cells and fresh tissues, and humanised mouse models to study immune response. Ultimately, randomised clinical trials for prospective therapies should use strong primary end-points such as overall survival comparing outcomes to the current standard therapies. At the clinical level, patients should be stratified based on strong data from genetic and cell biological preclinical analysis of mesothelioma cells.

The awareness of these gaps along with the increasing pace of knowledge regarding genomics and biology of mesothelioma will allow to multiply our chances of achieving a real improvement of the clinical outcomes for patients.

Acknowledgements: the authors would like to thank Patrick Brochard and Justine Gallet (Univ. Bordeaux, Bordeaux, France) and Eric Wasielewski (CHU Lille, Lille, France) for their help.

Conflict of interest: A. Scherpereel reports personal fees for advisory board work from AstraZeneca, BMS, MSD, Roche and Janssen, non-financial support for meeting attendance from BMS, MSD and Roche, institutional support for clinical trial participation from Astra-Zeneca/MedImmune, BMS, Verastem and Bayer, grants from BMS, outside the submitted work. I. Opitz has nothing to disclose. T. Berghmans has nothing to disclose. I. Psallidas works as a Medical Science Director for AstraZeneca, outside the submitted work; membership of the task force was resigned when this position became effective. M. Glatzer has nothing to disclose. D. Rigau works as methodologist for the European Respiratory Society. P. Astoul has nothing to disclose. S. Bölükbas has nothing to disclose. J. Boyd is an employee of the European Respiratory Society. J. Coolen has nothing to disclose. C. De Bondt has nothing to disclose. D. De Ruysscher reports grants from Bristol-Myers-Squibb AstraZeneca, Celgene, Roche/Genentech and Merck/ Pfizer, outside the submitted work. V. Durieux has nothing to disclose. C. Faivre-Finn has nothing to disclose. D. Fennell reports personal fees and 
non-financial support from BMS and MSD, non-financial support from Eli Lilly, Clovis, Bergen Bio and Pierre Fabre, grants, personal fees and non-financial support from Roche-Genentech, personal fees from Aldeyra, during the conduct of the study. F. Galateau-Salle has nothing to disclose. L. Greillier reports grants, personal fees and non-financial support from Roche and Novartis, personal fees and non-financial support from Pfizer, Bristol-Myers Squibb, Boehringer Ingelheim, AstraZeneca, Abbvie and MSD, outside the submitted work. M.A. Hoda has nothing to disclose. W. Klepetko has nothing to disclose. A. Lacourt has nothing to disclose. P. McElnay was employed by GlaxoSmithKline, outside the submitted work. N.A. Maskell has nothing to disclose. L. Mutti has nothing to disclose. J-C. Pairon reports grants from Santé Publique France Agency and French National Health Insurance (CNAM-TS), outside the submitted work. P. Van Schil has nothing to disclose. J.P. van Meerbeeck has nothing to disclose. D. Waller has nothing to disclose. W. Weder reports personal fees from AstraZeneca for advisory board work and lectures, grants and personal fees for lectures from Covidien. G. Cardillo has nothing to disclose. P.M. Putora reports grants from AstraZeneca and Celgene, outside the submitted work.

Support statement: This work was supported by the European Respiratory Society, European Society of Thoracic Surgeons, European Association for Cardio-Thoracic Surgery and the European Society for Radiotherapy and Oncology. Funding information for this article has been deposited with the Crossref Funder Registry.

This document was endorsed by the European Respiratory Society (ERS) on November 10, 2019, by the European Society of Thoracic Surgeons (ESTS) on November 7, 2019, by the European Association for Cardio-Thoracic Surgery (EACTS) on November 4, 2019 and by the European Society for Radiotherapy and Oncology (ESTRO) on February 17, 2020.

The guidelines published by the European Respiratory Society (ERS) incorporate data obtained from a comprehensive and systematic literature review of the most recent studies available at the time. Health professionals are encouraged to take the guidelines into account in their clinical practice. However, the recommendations issued by this guideline may not be appropriate for use in all situations. It is the individual responsibility of health professionals to consult other sources of relevant information, to make appropriate and accurate decisions in consideration of each patient's health condition and in consultation with that patient and the patient's caregiver where appropriate and/or necessary, and to verify rules and regulations applicable to drugs and devices at the time of prescription.

\section{References}

1 Bueno R, Stawiski EW, Goldstein LD, et al. Comprehensive genomic analysis of malignant pleural mesothelioma identifies recurrent mutations, gene fusions and splicing alterations. Nat Genet 2016; 48: 407-416.

2 Yap TA, Aerts JG, Popat S, et al. Novel insights into mesothelioma biology and implications for therapy. Nat Rev Cancer 2017; 17: 475-488.

3 Scherpereel A, Wallyn F, Albelda S, et al. Novel medical therapies for malignant pleural mesothelioma: a review. Lancet Oncol 2018; 19: e161-e172.

4 Woolhouse I, Bishop L, Darlison L, et al. British Thoracic Society Guideline for the investigation and management of malignant pleural mesothelioma. Thorax 2018; 73: Suppl. 1, i1-i30.

5 Kindler HL, Ismaila N, Armato SG 3rd, et al. Treatment of malignant pleural mesothelioma: American Society of Clinical Oncology clinical practice guideline. J Clin Oncol 2018; 36: 1343-1373.

6 National Comprehensive Cancer Network (NCCN). Malignant Pleural Mesothelioma Guidelines Version 2.2018 NCCN , 2018. www.nccn.org/professionals/physician_gls/pdf/mpm_blocks.pdf

7 Baas P, Fennell D, Kerr KM, et al. Malignant pleural mesothelioma: ESMO Clinical Practice Guidelines for diagnosis, treatment and follow-up. Ann Oncol 2015; 26: Suppl. 5, v31-v39.

8 Scherpereel A, Astoul P, Baas P, et al. Guidelines of the European Respiratory Society and the European Society of Thoracic Surgeons for the management of malignant pleural mesothelioma. Eur Respir J 2010; 35: 479-495.

9 Sackett DL. Evidence-Based Medicine: How to Practice and Teach EBM. New York, Edinburgh, Churchill Livingstone, 2000.

10 Bianchi C, Bianchi T. Global mesothelioma epidemic: trend and features. Indian J Occup Environ Med 2014; 18: 82-88.

11 Delgermaa V, Takahashi K, Park E-K, et al. Global mesothelioma deaths reported to the World Health Organization between 1994 and 2008. Bull World Health Organ 2011; 89: 716-724; 724A-724C.

12 Odgerel C-O, Takahashi K, Sorahan T, et al. Estimation of the global burden of mesothelioma deaths from incomplete national mortality data. Occup Environ Med 2017; 74: 851-858.

13 Røe OD, Stella GM. Malignant pleural mesothelioma: history, controversy and future of a manmade epidemic. Eur Respir Rev 2015; 24: 115-131.

14 Kameda T, Takahashi K, Kim R, et al. Asbestos: use, bans and disease burden in Europe. Bull World Health Organ 2014; 92: 790-797.

15 Soberg MJ, van Zandwijk N. Incidence of malignant mesothelioma in New Zealand and Australia: a global snapshot. N Z Med J 2015; 128: 68-71.

16 Le GV, Takahashi K, Park EK, et al. Asbestos use and asbestos-related diseases in Asia: past, present and future. Respirology 2011; 16: 767-775.

17 Fazzo L, De Santis M, Minelli G, et al. Pleural mesothelioma mortality and asbestos exposure mapping in Italy. Am J Ind Med 2012; 55: 11-24.

18 Marinaccio A, Binazzi A, Marzio DD, et al. Pleural malignant mesothelioma epidemic: incidence, modalities of asbestos exposure and occupations involved from the Italian National Register. Int J Cancer 2012; 130: 2146-2154.

19 Korda RJ, Clements MS, Armstrong BK, et al. Mesothelioma trends in the ACT and comparisons with the rest of Australia. Public Health Res Pract 2016; 26: 2641646.

20 Soeberg MJ, Creighton N, Currow DC, et al. Patterns in the incidence, mortality and survival of malignant pleural and peritoneal mesothelioma, New South Wales, 1972-2009. Aust N Z J Public Health 2016; 40: 255-262.

21 Soeberg MJ, Leigh J, Driscoll T, et al. Incidence and survival trends for malignant pleural and peritoneal mesothelioma, Australia, 1982-2009. Occup Environ Med 2016; 73: 187-194. 

from 1984 to 2007, and projections from 2008 to 2032. Am J Ind Med 2015; 58: 473-482.

23 Van den Borre L, Deboosere P. Asbestos in Belgium: an underestimated health risk. The evolution of mesothelioma mortality rates (1969-2009). Int J Occup Environ Health 2014; 20: 134-140.

24 Tomasson K, Gudmundsson G, Briem H, et al. Malignant mesothelioma incidence by nation-wide cancer registry: a population-based study. J Occup Med Toxicol 2016; 11: 37.

25 Schonfeld SJ, McCormack V, Rutherford MJ, et al. Regional variations in German mesothelioma mortality rates: 2000-2010. Cancer Causes Control 2014; 25: 615-624.

26 Lehnert M, Kraywinkel K, Heinze E, et al. Incidence of malignant mesothelioma in Germany 2009-2013. Cancer Causes Control 2017; 28: 97-105.

27 Zadnik V, Primic Zakelj M, Jarm K, et al. Time trends and spatial patterns in the mesothelioma incidence in Slovenia, 1961-2014. Eur J Cancer Prev 2017; 26: S191-S196.

28 Glynn ME, Keeton KA, Gaffney SH, et al. Ambient asbestos fiber concentrations and long-term trends in pleural mesothelioma incidence between urban and rural areas in the United States (1973-2012). Risk Anal 2018; 38: 454-471.

29 Henley SJ, Larson TC, Wu M, et al. Mesothelioma incidence in 50 states and the District of Columbia, United States, 2003-2008. Int J Occup Environ Health 2013; 19: 1-10.

30 Mokdad AH, Dwyer-Lindgren L, Fitzmaurice C, et al. Trends and patterns of disparities in cancer mortality among US counties, 1980-2014. JAMA 2017; 317: 388-406.

31 Helland A, Solberg S, Brustugun OT. Incidence and survival of malignant pleural mesothelioma in Norway: a population-based study of 1686 cases. J Thorac Oncol 2012; 7: 1858-1861.

32 Kielkowski D, Nelson G, Bello B, et al. Trends in mesothelioma mortality rates in South Africa: 1995-2007. Occup Environ Med 2011; 68: 547-549.

33 Jennings CJ, Walsh PM, Deady S, et al. Malignant pleural mesothelioma incidence and survival in the Republic of Ireland 1994-2009. Cancer Epidemiol 2014; 38: 35-41.

34 Le Stang N, Belot A, Gilg Soit Ilg A, et al. Evolution of pleural cancers and malignant pleural mesothelioma incidence in France between 1980 and 2005. Int J Cancer 2010; 126: 232-238.

35 Skammeritz E, Omland $\varnothing$, Hansen J, et al. Regional differences in incidence of malignant mesothelioma in Denmark. Dan Med J 2013; 60: A4592.

36 Järvholm B, Burdorf A. Emerging evidence that the ban on asbestos use is reducing the occurrence of pleura mesothelioma in Sweden. Scand J Public Health 2015; 43: 875-881.

37 Zhao J, Zuo T, Zheng R, et al. Epidemiology and trend analysis on malignant mesothelioma in China. Chin J Cancer Res 2017; 29: 361-368.

38 Algranti E, Saito CA, Carneiro APS, et al. The next mesothelioma wave: mortality trends and forecast to 2030 in Brazil. Cancer Epidemiol 2015; 39: 687-692.

39 Jung SH, Kim HR, Koh SB, et al. A decade of malignant mesothelioma surveillance in Korea. Am J Ind Med 2012; 55: 869-875.

40 Kwak KM, Paek D, Hwang SS, et al. Estimated future incidence of malignant mesothelioma in South Korea: projection from 2014 to 2033. PLoS One 2017; 12: e0183404.

41 Lee HY, Hyun SH, Lee KS, et al. Volume-based parameter of ${ }^{18}$ F-FDG PET/CT in malignant pleural mesothelioma: prediction of therapeutic response and prognostic implications. Ann Surg Oncol 2010; 17: 2787-2794.

42 Tse LA, Yu IT, Goggins W, et al. Are current or future mesothelioma epidemics in Hong Kong the tragic legacy of uncontrolled use of asbestos in the past? Environ Health Perspect 2010; 118: 382-386.

43 López-Abente G, García-Gómez M, Menéndez-Navarro A, et al. Pleural cancer mortality in Spain: time-trends and updating of predictions up to 2020. BMC Cancer 2013; 13: 528.

44 Lin RT, Takahashi K, Karjalainen A, et al. Ecological association between asbestos-related diseases and historical asbestos consumption: an international analysis. Lancet 2007; 369: 844-849.

45 Peto J, Decarli A, La Vecchia C, et al. The European mesothelioma epidemic. Br J Cancer 1999; 79: 666-672.

46 Trama A, Marcos-Gragera R, Sánchez Pérez MJ, et al. Data quality in rare cancers registration: the report of the RARECARE data quality study. Tumori 2017; 103: 22-32.

47 Berman DW, Crump KS. A meta-analysis of asbestos-related cancer risk that addresses fiber size and mineral type. Crit Rev Toxicol 2008; 38: Suppl. 1, 49-73.

48 Lanphear BP, Buncher CR. Latent period for malignant mesothelioma of occupational origin. J Occup Med 1992; 34: 718-721.

49 Aguilar-Madrid G, Robles-Perez E, Juárez-Pérez CA, et al. Case-control study of pleural mesothelioma in workers with social security in Mexico. Am J Ind Med 2010; 53: 241-251.

50 Rushton L, Bagga S, Bevan R, et al. Occupation and cancer in Britain. Br J Cancer 2010; 102 : 1428-1437.

51 Lacourt A, Gramond C, Rolland P, et al. Occupational and non-occupational attributable risk of asbestos exposure for malignant pleural mesothelioma. Thorax 2014; 69: 532-539.

52 Ferrante D, Mirabelli D, Tunesi S, et al. Pleural mesothelioma and occupational and non-occupational asbestos exposure: a case-control study with quantitative risk assessment. Occup Environ Med 2016; 73: 147-153.

53 Liu B, van Gerwen M, Bonassi S, et al. Epidemiology of environmental exposure and malignant mesothelioma. J Thorac Oncol 2017; 12: 1031-1045.

54 Marsh GM, Benson SM. Response to: 'Pleural mesothelioma and occupational and non-occupational asbestos exposure: a case-control study with quantitative risk assessment' by Ferrante et al. Occup Environ Med 2017; 74: $156-157$.

55 Baumann F, Maurizot P, Mangeas M, et al. Pleural mesothelioma in New Caledonia: associations with environmental risk factors. Environ Health Perspect 2011; 119: 695-700.

56 Berk S, Yalcin H, Dogan OT, et al. The assessment of the malignant mesothelioma cases and environmental asbestos exposure in Sivas province, Turkey. Environ Geochem Health 2014; 36: 55-64.

57 Bourgault $\mathrm{MH}$, Gagné M, Valcke M. Lung cancer and mesothelioma risk assessment for a population environmentally exposed to asbestos. Int J Hyg Environ Health 2014; 217: 340-346.

58 Corfiati M, Scarselli A, Binazzi A, et al. Epidemiological patterns of asbestos exposure and spatial clusters of incident cases of malignant mesothelioma from the Italian national registry. BMC Cancer 2015; 15: 286. 
D’Agostin F, de Michieli P, Negro C. Pleural mesothelioma in household members of asbestos-exposed workers in Friuli Venezia Giulia, Italy. Int J Occup Med Environ Health 2017; 30: 419-431.

60 Driece HAL, Siesling S, Swuste PH, et al. Assessment of cancer risks due to environmental exposure to asbestos. J Expo Sci Environ Epidemiol 2010; 20: 478-485.

61 Fazzo L, Menegozzo S, Soggiu ME, et al. Mesothelioma incidence in the neighbourhood of an asbestos-cement plant located in a national priority contaminated site. Ann Ist Super Sanita 2014; 50: 322-327.

62 Gogali A, Manda-Stachouli C, Ntzani EE, et al. Malignant mesothelioma in Metsovo, Greece, from domestic use of asbestos: 30 years later. Eur Respir J 2012; 39: 217-219.

63 Goldberg S, Rey G, Luce D, et al. Possible effect of environmental exposure to asbestos on geographical variation in mesothelioma rates. Occup Environ Med 2010: 67: 417-421.

64 Goswami E, Craven V, Dahlstrom DL, et al. Domestic asbestos exposure: a review of epidemiologic and exposure data. Int J Environ Res Public Health 2013; 10: 5629-5670.

65 Langhoff MD, Kragh-Thomsen MB, Stanislaus S, et al. Almost half of women with malignant mesothelioma were exposed to asbestos at home through their husbands or sons. Dan Med J 2014; 61: A4902.

66 López-Abente G, Fernández-Navarro P, Boldo E, et al. Industrial pollution and pleural cancer mortality in Spain. Sci Total Environ 2012; 424: 57-62.

67 Marinaccio A, Binazzi A, Bonafede M, et al. Malignant mesothelioma due to non-occupational asbestos exposure from the Italian national surveillance system (ReNaM): epidemiology and public health issues. Occup Environ Med 2015; 72: 648-655.

68 Mensi C, Riboldi L, De Matteis S, et al. Impact of an asbestos cement factory on mesothelioma incidence: global assessment of effects of occupational, familial, and environmental exposure. Environ Int 2015; 74: 191-199.

69 Mirabelli D, Cavone D, Merler E, et al. Non-occupational exposure to asbestos and malignant mesothelioma in the Italian National Registry of Mesotheliomas. Occup Environ Med 2010; 67: 792-794.

70 Olsen NJ, Franklin PJ, Reid A, et al. Increasing incidence of malignant mesothelioma after exposure to asbestos during home maintenance and renovation. Med J Aust 2011; 195: 271-274.

71 Reid A, Franklin P, Olsen N, et al. All-cause mortality and cancer incidence among adults exposed to blue asbestos during childhood. Am J Ind Med 2013; 56: 133-145.

72 Salerno C, Berchialla P, Palin LA, et al. Cancer morbidity of residents living near an oil refinery plant in North-West Italy. Int J Environ Health Res 2013; 23: 342-351.

73 Tarrés J, Albertí C, Martínez-Artés X, et al. Pleural mesothelioma in relation to meteorological conditions and residential distance from an industrial source of asbestos. Occup Environ Med 2013; 70: 588-590.

74 Wei B, Jia X, Ye B, et al. Impacts of land use on spatial distribution of mortality rates of cancers caused by naturally occurring asbestos. J Expo Sci Environ Epidemiol 2012; 22: 516-521.

75 Metintas M, Hillerdal G, Metintas S, et al. Endemic malignant mesothelioma: exposure to erionite is more important than genetic factors. Arch Environ Occup Health 2010; 65: 86-93.

76 Ortega-Guerrero MA, Carrasco-Núñez G. Environmental occurrence, origin, physical and geochemical properties, and carcinogenic potential of erionite near San Miguel de Allende, Mexico. Environ Geochem Health 2014; 36: 517-529.

77 Dunning KK, Adjei S, Levin L, et al. Mesothelioma associated with commercial use of vermiculite containing Libby amphibole. J Occup Environ Med 2012; 54: 1359-1363.

78 Larson TC, Antao VC, Bove FJ. Vermiculite worker mortality: estimated effects of occupational exposure to Libby amphibole. J Occup Environ Med 2010; 52: 555-560.

79 Moolgavkar SH, Turim J, Alexander DD, et al. Potency factors for risk assessment at Libby, Montana. Risk Anal 2010; 30: 1240-1248.

80 Bruno C, Tumino R, Fazzo L, et al. Incidence of pleural mesothelioma in a community exposed to fibres with fluoro-edenitic composition in Biancavilla (Sicily, Italy). Ann Ist Super Sanita 2014; 50: 111-118.

81 Conti S, Minelli G, Manno V, et al. Health impact of the exposure to fibres with fluoro-edenitic composition on the residents in Biancavilla (Sicily, Italy): mortality and hospitalization from current data. Ann Ist Super Sanita 2014; 50: 127-132.

82 Abakay A, Tanrikulu AC, Ayhan M, et al. High-risk mesothelioma relation to meteorological and geological condition and distance from naturally occurring asbestos. Environ Health Prev Med 2016; 21: 82-90.

83 Bayram M, Dongel I, Bakan ND, et al. High risk of malignant mesothelioma and pleural plaques in subjects born close to ophiolites. Chest 2013; 143: 164-171.

84 Baumann F, Flores E, Napolitano A, et al. Mesothelioma patients with germline BAP1 mutations have 7-fold improved long-term survival. Carcinogenesis 2015; 36: 76-81.

85 Allen EM, Alexander BH, MacLehose RF, et al. Mortality experience among Minnesota taconite mining industry workers. Occup Environ Med 2014; 71: 744-749.

86 Lambert CS, Alexander BH, Ramachandran G, et al. A case-control study of mesothelioma in Minnesota iron ore (taconite) miners. Occup Environ Med 2016; 73: 103-109.

87 Finkelstein MM. Malignant mesothelioma incidence among talc miners and millers in New York State. Am J Ind Med 2012; 55: 863-868.

88 Walker AM, Maxim LD, Utell MJ. Are airborne refractory ceramic fibers similar to asbestos in their carcinogenicity? Inhal Toxicol 2012; 24: 416-424.

89 LeMasters G, Lockey JE, Hilbert TJ, et al. A 30-year mortality and respiratory morbidity study of refractory ceramic fiber workers. Inhal Toxicol 2017; 29: 462-470.

90 Grosse Y, Loomis D, Guyton KZ, et al. Carcinogenicity of fluoro-edenite, silicon carbide fibres and whiskers, and carbon nanotubes. Lancet Oncol 2014; 15: 1427-1428.

91 Overall evaluations of carcinogenicity: an updating of IARC Monographs volumes 1 to 42. IARC Monogr Eval Carcinog Risks Hum Suppl 1987; 7: 1-440.

92 International Agency for Cancer Research (IARC). IARC Monographs on the Evaluation of Carcinogenic Risks to Humans Silica and Some Silicates, Volume 42. Reviews of Human Carcinogens. IARC Monographs on the Evaluation of Carcinogenic Risks to Humans. International Agency for Research on Cancer, World Health Organization, 1987.

93 International Agency for Cancer Research (IARC). IARC Monographs - Arsenic, Metals, Fibres and Dusts, Volume 100 C. Reviews of Human Carcinogens. IARC Monographs on the Evaluation of Carcinogenic Risks to Humans. International Agency for Research on Cancer, World Health Organization, 2012. 

and whiskers, and single-walled and multi-walled carbon nanotub, Volume 111. Reviews of Human Carcinogens. IARC Monographs on the Evaluation of Carcinogenic Risks to Humans. International Agency for Research on Cancer, World Health Organization, 2014.

95 Ortega-Guerrero MA, Carrasco-Núñez G, Barragán-Campos H, et al. High incidence of lung cancer and malignant mesothelioma linked to erionite fibre exposure in a rural community in Central Mexico. Occup Environ Med 2015; 72: 216-218.

96 Van Gosen BS, Blitz TA, Plumlee GS, et al. Geologic occurrences of erionite in the United States: an emerging national public health concern for respiratory disease. Environ Geochem Health 2013; 35: 419-430.

97 Carbone M, Baris YI, Bertino P, et al. Erionite exposure in North Dakota and Turkish villages with mesothelioma. Proc Natl Acad Sci USA 2011; 108: 13618-13623.

98 Lacourt A, Gramond C, Audignon S, et al. Pleural mesothelioma and occupational coexposure to asbestos, mineral wool, and silica. Am J Respir Crit Care Med 2013; 187: 977-982.

99 Pintos J, Parent ME, Case BW, et al. Risk of mesothelioma and occupational exposure to asbestos and man-made vitreous fibers: evidence from two case-control studies in Montreal, Canada. J Occup Environ Med 2009; 51: $1177-1184$

100 Rödelsperger K, Jöckel KH, Pohlabeln H, et al. Asbestos and man-made vitreous fibers as risk factors for diffuse malignant mesothelioma: results from a German hospital-based case-control study. Am J Ind Med 2001; 39: $262-275$.

101 Suzui M, Futakuchi M, Fukamachi K, et al. Multiwalled carbon nanotubes intratracheally instilled into the rat lung induce development of pleural malignant mesothelioma and lung tumors. Cancer Sci 2016; 107: 924-935.

102 Fukushima S, Kasai T, Umeda Y, et al. Carcinogenicity of multi-walled carbon nanotubes: challenging issue on hazard assessment. J Occup Health 2018; 60: 10-30.

103 Ji J, Sundquist J, Sundquist K. Incidence and familial risk of pleural mesothelioma in Sweden: a national cohort study. Eur Respir J 2016; 48: 873-879.

104 Ascoli V, Romeo E, Carnovale Scalzo C, et al. Familial malignant mesothelioma: a population-based study in central Italy (1980-2012). Cancer Epidemiol 2014; 38: 273-278.

105 de Klerk N, Alfonso H, Olsen N, et al. Familial aggregation of malignant mesothelioma in former workers and residents of Wittenoom, Western Australia. Int J Cancer 2013; 132: 1423-1428.

106 Testa JR, Cheung M, Pei J, et al. Germline BAP1 mutations predispose to malignant mesothelioma. Nat Genet 2011; 43: 1022-1025.

107 Cheung M, Talarchek J, Schindeler K, et al. Further evidence for germline BAP1 mutations predisposing to melanoma and malignant mesothelioma. Cancer Genet 2013; 206: 206-210.

108 Cheung M, Kadariya Y, Talarchek J, et al. Germline BAP1 mutation in a family with high incidence of multiple primary cancers and a potential gene-environment interaction. Cancer Lett 2015; 369: 261-265.

109 Cheung M, Testa JR. BAP1, a tumor suppressor gene driving malignant mesothelioma. Transl Lung Cancer Res 2017; 6: 270-278.

110 Carbone M, Yang H, Pass HI, et al. BAP1 and cancer. Nat Rev Cancer 2013; 13: 153-159.

111 Betti M, Aspesi A, Biasi A, et al. CDKN2A and BAP1 germline mutations predispose to melanoma and mesothelioma. Cancer Lett 2016; 378: 120-130.

112 Andujar P, Lacourt A, Brochard P, et al. Five years update on relationships between malignant pleural mesothelioma and exposure to asbestos and other elongated mineral particles. J Toxicol Environ Health B Crit Rev 2016; 19: 151-172.

113 Andujar P, Pairon J-C, Renier A, et al. Differential mutation profiles and similar intronic TP53 polymorphisms in asbestos-related lung cancer and pleural mesothelioma. Mutagenesis 2013; 28: 323-331.

114 Betti M, Ferrante D, Padoan M, et al. XRCC1 and ERCC1 variants modify malignant mesothelioma risk: a case-control study. Mutat Res 2011; 708: 11-20.

115 Betti M, Casalone E, Ferrante D, et al. Germline mutations in DNA repair genes predispose asbestos-exposed patients to malignant pleural mesothelioma. Cancer Lett 2017; 405: 38-45.

116 Borelli V, Moura RR, Trevisan E, et al. NLRP1 and NLRP3 polymorphisms in mesothelioma patients and asbestos exposed individuals a population-based autopsy study from North East Italy. Infect Agents Cancer 2015; 10: 26.

117 Girardelli M, Maestri I, Rinaldi RR, et al. NLRP1 polymorphisms in patients with asbestos-associated mesothelioma. Infect Agent Cancer 2012; 7: 25.

118 Cadby G, Mukherjee S, Musk AWB, et al. A genome-wide association study for malignant mesothelioma risk. Lung Cancer 2013; 82: 1-8.

119 Matullo G, Guarrera S, Betti M, et al. Genetic variants associated with increased risk of malignant pleural mesothelioma: a genome-wide association study. PLoS One 2013; 8: e61253.

120 Tunesi S, Ferrante D, Mirabelli D, et al. Gene-asbestos interaction in malignant pleural mesothelioma susceptibility. Carcinogenesis 2015; 36: 1129-1135.

121 Panou V, Gadiraju M, Wolin A, et al. Frequency of germline mutations in cancer susceptibility genes in malignant mesothelioma. J Clin Oncol 2018; 36: 2863-2871.

122 Hassan R, Morrow B, Walsh $\mathrm{T}$, et al. Inherited predisposition to malignant mesothelioma (MM) due to mutations in DNA repair genes. J Clin Oncol 2018; 36: Suppl. 15, 8504.

123 Peterson JT, Greenberg SD, Buffler PA. Non-asbestos-related malignant mesothelioma. A review. Cancer 1984; 54: 951-960.

124 Farioli A, Ottone M, Morganti AG, et al. Radiation-induced mesothelioma among long-term solid cancer survivors: a longitudinal analysis of SEER database. Cancer Med 2016; 5: 950-959.

125 International Agency for Cancer Research (IARC). IARC Monographs - Malaria and Some Polyomaviruses (SV40, BK, JC, and Merkel Cell Viruses), Volume 104. Reviews of Human Carcinogens. IARC Monographs on the Evaluation of Carcinogenic Risks to Humans. International Agency for Research on Cancer, World Health Organization, 2014.

126 Bianchi C, Brollo A, Ramani L, et al. Pleural plaques as risk indicators for malignant pleural mesothelioma: a necropsy-based study. Am J Ind Med 1997; 32: 445-449. 
Banks DE, Shi R, McLarty J, et al. American College of Chest Physicians consensus statement on the respiratory health effects of asbestos. Results of a Delphi study. Chest 2009; 135: 1619-1627.

Maxim LD, Niebo R, Utell MJ. Are pleural plaques an appropriate endpoint for risk analyses? Inhal Toxicol 2015; 27: 321-334.

Pairon J-C, Laurent F, Rinaldo M, et al. Pleural plaques and the risk of pleural mesothelioma. J Natl Cancer Inst 2013; 105: 293-301.

Robinson BW, Creaney J, Lake R, et al. Mesothelin-family proteins and diagnosis of mesothelioma. Lancet 2003; 362: 1612-1616.

Hollevoet K, Nackaerts K, Thimpont J, et al. Diagnostic performance of soluble mesothelin and megakaryocyte potentiating factor in mesothelioma. Am J Respir Crit Care Med 2010; 181: 620-625.

Bayram M, Dongel I, Akbas A, et al. Serum biomarkers in patients with mesothelioma and pleural plaques and healthy subjects exposed to naturally occurring asbestos. Lung 2014; 192: 197-203.

Creaney J, Dick IM, Meniawy TM, et
mesothelioma. Thorax 2014; 69: 895-902.

Luo L, Shi HZ, Liang QL, et al. Diagnostic value of soluble mesothelin-related peptides for malignant mesothelioma: a meta-analysis. Respir Med 2010; 104: 149-156.

Hollevoet K, Reitsma JB, Creaney J, et al. Serum mesothelin for diagnosing malignant pleural mesothelioma: an individual patient data meta-analysis. J Clin Oncol 2012; 30: 1541-1549.

I Med 2005; 353: 1564-1573.

Hu ZD, Liu XF, Liu XC, et al. Diagnostic accuracy of osteopontin for malignant pleural mesothelioma: a systematic review and meta-analysis. Clin Chim Acta 2014; 433: 44-48.

Pass HI, Levin SM, Harbut MR, et al. Fibulin-3 as a blood and effusion biomarker for pleural mesothelioma. N Engl J Med 2012; 367: 1417-1427.

Pei D, Li Y, Liu X, et al. Diagnostic and prognostic utilities of humoral fibulin-3 in malignant pleural mesothelioma: evidence from a meta-analysis. Oncotarget 2017; 8: 13030-13038.

Kirschner MB, Cheng YY, Badrian B, et al. Increased circulating miR-625-3p: a potential biomarker for patients with malignant pleural mesothelioma. J Thorac Oncol 2012; 7: 1184-1191.

Santarelli L, Staffolani S, Strafella E, et al. Combined circulating epigenetic markers to improve mesothelin performance in the diagnosis of malignant mesothelioma. Lung Cancer 2015; 90: 457-464.

Bononi I, Comar M, Puozzo A, et al. Circulating microRNAs found dysregulated in ex-exposed asbestos workers and pleural mesothelioma patients as potential new biomarkers. Oncotarget 2016; 7: 82700-82711.

Weber DG, Gawrych K, Casjens S, et al. Circulating miR-132-3p as a candidate diagnostic biomarker for malignant mesothelioma. Dis Markers 2017; 2017: 9280170.

Napolitano A, Antoine DJ, Pellegrini L, et al. HMGB1 and its hyperacetylated isoform are sensitive and specific serum biomarkers to detect asbestos exposure and to identify mesothelioma patients. Clin Cancer Res 2016; 22: 3087-3096.

Ying S, Jiang Z, He X, et al. Serum HMGB1 as a potential biomarker for patients with asbestos-related diseases. Dis Markers 2017; 2017: 5756102.

Onda M, Nagata S, Ho M, et al. Megakaryocyte potentiation factor cleaved from mesothelin precursor is a useful tumor marker in the serum of patients with mesothelioma. Clin Cancer Res 2006; 12: 4225-4231.

Ostroff RM, Mehan MR, Stewart A, et al. Early detection of malignant pleural mesothelioma in asbestos-exposed individuals with a noninvasive proteomics-based surveillance tool. PLoS One 2012; 7: e46091.

Watzka SB, Posch F, Pass HI, et al. Serum concentration of integrin-linked kinase in malignant pleural mesothelioma and after asbestos exposure. Eur J Cardiothorac Surg 2013; 43: 940-945.

$\mathrm{Xu}$ J, Alexander DB, Iigo M, et al. Chemokine (C-C motif) ligand 3 detection in the serum of persons exposed to asbestos: a patient-based study. Cancer Sci 2015; 106: 825-832.

Demir M, Kaya H, Taylan M, et al. Evaluation of new biomarkers in the prediction of malignant mesothelioma in subjects with environmental asbestos exposure. Lung 2016; 194: 409-417. malignant mesothelioma 4-10 years in advance of clinical symptoms. Clin Proteomics 2016; 13: 2.

Johnen G, Gawrych K, Raiko I, et al. Calretinin as a blood-based biomarker for mesothelioma. BMC Cancer 2017; 17: 386

Cristaudo A, Bonotti A, Simonini S, et al. Soluble markers for diagnosis of malignant pleural mesothelioma. Biomark Med 2011; 5: 261-273.

Pantazopoulos I, Boura P, Xanthos T, et al. Effectiveness of mesothelin family proteins and osteopontin for malignant mesothelioma. Eur Respir J 2013; 41: 706-715.

Chen Z, Gaudino G, Pass HI, et al. Diagnostic and prognostic biomarkers for malignant mesothelioma: an update. Transl Lung Cancer Res 2017; 6: 259-269.

Creaney J, Robinson BWS. Malignant mesothelioma biomarkers: from discovery to use in clinical practice for diagnosis, monitoring, screening, and treatment. Chest 2017; 152: 143-149.

Sun HH, Vaynblat A, Pass HI. Diagnosis and prognosis-review of biomarkers for mesothelioma. Ann Transl Med 2017; 5: 244.

Park EK, Sandrini A, Yates DH, et al. Soluble mesothelin-related protein in an asbestos-exposed population: the Dust Diseases Board Cohort Study. Am J Respir Crit Care Med 2008; 178: 832-837.

Hollevoet K, Van Cleemput J, Thimpont J, et al. Serial measurements of mesothelioma serum biomarkers in asbestos-exposed individuals: a prospective longitudinal cohort study. J Thorac Oncol 2011; 6: 889-895.

160 Gube M, Taeger D, Weber DG, et al. Performance of biomarkers SMRP, CA125, and CYFRA 21-1 as potential tumor markers for malignant mesothelioma and lung cancer in a cohort of workers formerly exposed to asbestos. Arch Toxicol 2011; 85: 185-192.

161 Felten MK, Khatab K, Knoll L, et al. Changes of mesothelin and osteopontin levels over time in formerly asbestos-exposed power industry workers. Int Arch Occup Environ Health 2014; 87: 195-204.

Filiberti R, Marroni P, Spigno F, et al. Is soluble mesothelin-related protein an upfront predictive marker of pleural mesothelioma? A prospective study on Italian workers exposed to asbestos. Oncology 2014; 86: 33-43. 
Hirohashi T, Igarashi $\mathrm{K}$, Abe $\mathrm{M}$, et al. Retrospective analysis of large-scale research screening of construction workers for the early diagnosis of mesothelioma. Mol Clin Oncol 2014; 2: 26-30.

Creaney J, Olsen NJ, Brims F, et al. Serum mesothelin for early detection of asbestos-induced cancer malignant mesothelioma. Cancer Epidemiol Biomarkers Prev 2010; 19: 2238-2246.

Kirkham TL, Koehoorn MW, McLeod CB, et al. Surveillance of mesothelioma and workers' compensation in British Columbia, Canada. Occup Environ Med 2011; 68: 30-35.

García-Gómez M, Menéndez-Navarro A, López RC. Asbestos-related occupational cancers compensated under the Spanish National Insurance System, 1978-2011. Int J Occup Environ Health 2015; 21: 31-39.

Chamming's S, Clin B, Brochard P, et al. Compensation of pleural mesothelioma in France: data from the French National Mesothelioma Surveillance Programme. Am J Ind Med 2013; 56: 146-154.

Poe RH, Israel RH, Utell MJ, et al. Sensitivity, specificity, and predictive values of closed pleural biopsy. Arch Intern Med 1984; 144: 325-328.

Von Hoff DD, LiVolsi V. Diagnostic reliability of needle biopsy of the parietal pleura. A review of 272 biopsies. Am J Clin Pathol 1975; 64: 200-203.

Lancet 1958; 2: 1349-1353.

Tomlinson JR, Sahn SA. Invasive procedures in the diagnosis of pleural disease. Semin Respir Crit Care Med 1987; 9: 30-36.

Escudero Bueno C, García Clemente M, Cuesta Castro B, et al. Cytologic and bacteriologic analysis of fluid and pleural biopsy specimens with Cope's needle. Study of 414 patients. Arch Intern Med 1990; 150: 1190-1194.

Heilo A, Stenwig AE, Solheim OP. Malignant pleural mesothelioma: US-guided histologic core-needle biopsy. Radiology 1999; 211: 657-659.

Diacon AH, Schuurmans MM, Theron J, et al. Safety and yield of ultrasound-assisted transthoracic biopsy performed by pulmonologists. Respiration 2004; 71: 519-522.

Maskell NA, Gleeson FV, Davies RJ. Standard pleural biopsy versus CT-guided cutting-needle biopsy for diagnosis of malignant disease in pleural effusions: a randomised controlled trial. Lancet 2003; 361: 1326-1330.

Hallifax RJ, Corcoran JP, Ahmed A, et al. Physician-based ultrasound-guided biopsy for diagnosing pleural disease. Chest 2014; 146: 1001-1006.

Zahid I, Sharif S, Routledge T, et al. What is the best way to diagnose and stage malignant pleural mesothelioma? Interact Cardiovasc Thorac Surg 2011; 12: 254-259.

Greillier L, Cavailles A, Fraticelli A, et al. Accuracy of pleural biopsy using thoracoscopy for the diagnosis of histologic subtype in patients with malignant pleural mesothelioma. Cancer 2007; 110: 2248-2252.

Pinelli V, Laroumagne S, Sakr L, et al. Pleural fluid cytological yield and visceral pleural invasion in patients with epithelioid malignant pleural mesothelioma. J Thorac Oncol 2012; 7: 595-598.

Rahman NM, Ali NJ, Brown G, et al. Local anaesthetic thoracoscopy: British Thoracic Society Pleural Disease Guideline 2010. Thorax 2010; 65: Suppl. 2, ii54-ii60.

Maturu VN, Dhooria S, Bal A, et al. Role of medical thoracoscopy and closed-blind pleural biopsy in undiagnosed exudative pleural effusions: a single-center experience of 348 patients. J Bronchology Interv Pulmonol 2015; 22: 121-129.

Son HS, Lee SH, Darlong LM, et al. Is there a role for a needle thoracoscopic pleural biopsy under local anesthesia for pleural effusions? Korean J Thorac Cardiovasc Surg 2014; 47: 124-128.

Haridas N, Suraj KP, Rajagopal TP, et al. Medical thoracoscopy vs closed pleural biopsy in pleural effusions: a randomized controlled study. J Clin Diagn Res 2014: 8: MC01-MC04.

Mohamed EE, Talaat IM, Abd Alla AEDAA, et al. Diagnosis of exudative pleural effusion using ultrasound guided versus medical thoracoscopic pleural biopsy. Egypt J Chest Dis Tuberc 2013; 62: 607-615.

Bueno R, Opitz I, IASLC Mesothelioma Taskforce. Surgery in malignant pleural mesothelioma. J Thorac Oncol 2018; 13: 1638-1654.

Bölükbas S, Eberlein M, Kudelin N, et al. Factors predicting poor survival after lung-sparing radical pleurectomy of IMIG stage III malignant pleural mesothelioma. Eur J Cardiothorac Surg 2013; 44: 119-123.

Richards WG, Godleski JJ, Yeap BY, et al. Proposed adjustments to pathologic staging of epithelial malignant pleural mesothelioma based on analysis of 354 cases. Cancer 2010; 116: 1510-1517.

Yanagawa J, Rusch V. Surgical management of malignant pleural mesothelioma. Thorac Surg Clin 2013; 23: 73-87.

Bölükbas S, Eberlein M, Schirren J. Video-atlas of radical pleurectomy for malignant pleural mesothelioma. Ann Cardiothorac Surg 2012; 1: 534-536.

Bölükbas S, Eberlein M, Schirren J. Pleurectomy/decortication for the treatment of malignant pleural mesothelioma. In: Kuzdzal J, ed. ESTS Textbook of Thoracic Surgery. Cracow, Medicina Praktyczna, 2014; pp. 313-320.

Galateau-Salle F, Churg A, Roggli V, et al. The 2015 World Health Organization classification of tumors of the pleura: advances since the 2004 classification. J Thorac Oncol 2016; 11: 142-154.

the rat parotid gland. Arch Oral Biol 1977; 22: 261-267.

3 Husain AN, Colby TV, Ordóñez NG, et al. Guidelines for pathologic diagnosis of malignant mesothelioma. 2017 update of the consensus statement from the International Mesothelioma Interest Group. Arch Pathol Lab Med 2018; 142: 89-108.

94 Churg A, Roggli V, Galateau-Salle F. Tumours of the pleura. In: Travis WD, Brambilla E, Burke AP, et al., eds. WHO Classification of Tumours of the Lung, Pleura, Thymus, and Heart. Lyon, International Agency for Research on Cancer, 2015.

Arnold DT, De Fonseka D, Perry S, et al. Investigating unilateral pleural effusions: the role of cytology. Eur Respir J 2018; 52: 1801254.

Adams RF, Gray W, Davies RJ, et al. Percutaneous image-guided cutting needle biopsy of the pleura in the diagnosis of malignant mesothelioma. Chest 2001; 120: 1798-1802.

Churg A, Nabeshima K, Ali G, et al. Highlights of the 14th International Mesothelioma Interest Group meeting: pathologic separation of benign from malignant mesothelial proliferations and histologic/molecular analysis of malignant mesothelioma subtypes. Lung Cancer 2018; 124: 95-101. 

FISH in the diagnosis of malignant mesothelioma in effusion cytology specimens. Am J Surg Pathol 2016; 40: 120-126.

199 Nicholson AG, Sauter JL, Nowak AK, et al. EURACAN/IASLC proposals for updating the histologic classification of pleural mesothelioma: towards a more multidisciplinary approach. J Thorac Oncol 2020; 15: 29-49.

200 Marchevsky AM, LeStang N, Hiroshima K, et al. The differential diagnosis between pleural sarcomatoid mesothelioma and spindle cell/pleomorphic (sarcomatoid) carcinomas of the lung: evidence-based guidelines from the International Mesothelioma Panel and the MESOPATH National Reference Center. Hum Pathol 2017; 67: $160-168$.

201 Sheffield BS, Lorette J, Shen Y, et al. Immunohistochemistry for NF2, LATS1/2, and YAP/TAZ fails to separate benign from malignant mesothelial proliferations. Arch Pathol Lab Med 2016; 140: 391

202 Rusch VW, Giroux D, Kennedy C, et al. Initial analysis of the international association for the study of lung cancer mesothelioma database. J Thorac Oncol 2012; 7: 1631-1639.

203 Pass H, Giroux D, Kennedy C, et al. The IASLC Mesothelioma Staging Project: improving staging of a rare disease through international participation. J Thorac Oncol 2016; 11: 2082-2088.

204 Nowak AK, Chansky K, Rice DC, et al. The IASLC Mesothelioma Staging Project: proposals for revisions of the $\mathrm{T}$ descriptors in the forthcoming eighth edition of the TNM classification for pleural mesothelioma. $J$ Thorac Oncol 2016; 11: 2089-2099.

205 Rusch VW, Chansky K, Kindler HL, et al. The IASLC Mesothelioma Staging Project: proposals for the M descriptors and for revision of the TNM stage groupings in the forthcoming (eighth) edition of the TNM classification for mesothelioma. J Thorac Oncol 2016; 11: 2112-2119.

206 Rice D, Chansky K, Nowak A, et al. The IASLC Mesothelioma Staging Project: proposals for revisions of the N descriptors in the forthcoming eighth edition of the TNM classification for pleural mesothelioma. J Thorac Oncol 2016; 11: 2100-2111.

207 Pilling JE, Stewart DJ, Martin-Ucar AE, et al. The case for routine cervical mediastinoscopy prior to radical surgery for malignant pleural mesothelioma. Eur J Cardiothorac Surg 2004; 25: 497-501.

208 Sugarbaker DJ, Richards WG, Bueno R. Extrapleural pneumonectomy in the treatment of epithelioid malignant pleural mesothelioma: novel prognostic implications of combined N1 and N2 nodal involvement based on experience in 529 patients. Ann Surg 2014; 260: 577-580.

209 Kircheva DY, Husain AN, Watson S, et al. Specimen weight and volume: important predictors of survival in malignant pleural mesothelioma. Eur J Cardiothorac Surg 2016; 49: 1642-1647.

210 Edwards JG, Stewart DJ, Martin-Ucar A, et al. The pattern of lymph node involvement influences outcome after extrapleural pneumonectomy for malignant mesothelioma. J Thorac Cardiovasc Surg 2006; 131: 981-987.

211 Rusch VW, Gill R, Mitchell A, et al. A multicenter study of volumetric computed tomography for staging malignant pleural mesothelioma. Ann Thorac Surg 2016; 102: 1059-1066.

212 Erasmus JJ, Truong MT, Smythe WR, et al. Integrated computed tomography-positron emission tomography in patients with potentially resectable malignant pleural mesothelioma: staging implications. J Thorac Cardiovasc Surg 2005; 129: 1364-1370.

213 Billé A, Krug LM, Woo KM, et al. Contemporary analysis of prognostic factors in patients with unresectable malignant pleural mesothelioma. J Thorac Oncol 2016; 11: 249-255.

214 Martini K, Meier A, Opitz I, et al. Diagnostic accuracy of sequential co-registered PET+MR in comparison to PET/CT in local thoracic staging of malignant pleural mesothelioma. Lung Cancer 2016; 94: 40-45.

215 Gill RR, Umeoka S, Mamata H, et al. Diffusion-weighted MRI of malignant pleural mesothelioma: preliminary assessment of apparent diffusion coefficient in histologic subtypes. AJR Am J Roentgenol 2010; 195: W125-W130.

216 Chamberlain MH, Fareed K, Nakas A, et al. Video-assisted cervical thoracoscopy: a novel approach for diagnosis, staging and pleurodesis of malignant pleural mesothelioma. Eur J Cardiothorac Surg 2008; 34: $200-203$.

217 Rice DC, Steliga MA, Stewart J, et al. Endoscopic ultrasound-guided fine needle aspiration for staging of malignant pleural mesothelioma. Ann Thorac Surg 2009; 88: 862-868.

218 Nakas A, Waller D, Lau K, et al. The new case for cervical mediastinoscopy in selection for radical surgery for malignant pleural mesothelioma. Eur J Cardiothorac Surg 2012; 42: 72-76.

219 Zielinski M, Hauer J, Hauer L, et al. Staging algorithm for diffuse malignant pleural mesothelioma. Interact Cardiovasc Thorac Surg 2010; 10: 185-189.

220 Alvarez JM, Hasani A, Segal A, et al. Bilateral thoracoscopy, mediastinoscopy and laparoscopy, in addition to CT, MRI and PET imaging, are essential to correctly stage and treat patients with mesothelioma prior to trimodality therapy. ANZ J Surg 2009; 79: 734-738.

221 Pass HI, Giroux D, Kennedy C, et al. Supplementary prognostic variables for pleural mesothelioma: a report from the IASLC staging committee. J Thorac Oncol 2014; 9: 856-864.

222 Curran D, Sahmoud T, Therasse P, et al. Prognostic factors in patients with pleural mesothelioma: the European Organization for Research and Treatment of Cancer experience. J Clin Oncol 1998; 16: 145-152.

223 Herndon JE, Green MR, Chahinian AP, et al. Factors predictive of survival among 337 patients with mesothelioma treated between 1984 and 1994 by the Cancer and Leukemia Group B. Chest 1998; 113: 723-731.

224 Opitz I, Friess M, Kestenholz P, et al. A new prognostic score supporting treatment allocation for multimodality therapy for malignant pleural mesothelioma: a review of 12 years' experience. J Thorac Oncol 2015; 10: 1634-1641.

225 Brims FJ, Meniawy TM, Duffus I, et al. A novel clinical prediction model for prognosis in malignant pleural mesothelioma using decision tree analysis. J Thorac Oncol 2016; 11: 573-582.

226 Psallidas I, Kanellakis NI, Gerry S, et al. Development and validation of response markers to predict survival and pleurodesis success in patients with malignant pleural effusion (PROMISE): a multicohort analysis. Lancet Onco 2018; 19: 930-939.

227 Ben Bouazza Y, Van Meerbeeck JP. The use of patient-reported outcome measures (PROMs) in the management of malignant pleural mesothelioma: a descriptive literature survey. Transl Lung Cancer Res 2018; 7: 507-515.

228 Rintoul RC, Ritchie AJ, Edwards JG, et al. Efficacy and cost of video-assisted thoracoscopic partial pleurectomy versus talc pleurodesis in patients with malignant pleural mesothelioma (MesoVATS): an open-label, randomised, controlled trial. Lancet 2014; 384: 1118-1127. 
Rice D, Rusch V, Pass H, et al. Recommendations for uniform definitions of surgical techniques for malignant pleural mesothelioma: a consensus report of the International Association for the Study of Lung Cancer International Staging Committee and the International Mesothelioma Interest Group. J Thorac Oncol 2011; 6: 1304-1312.

230 Saddoughi SA, Abdelsattar ZM, Blackmon SH. National trends in the epidemiology of malignant pleural mesothelioma: a national cancer data base study. Ann Thorac Surg 2018; 105: 432-437.

231 Nelson DB, Rice DC, Niu J, et al. Predictors of trimodality therapy and trends in therapy for malignant pleural mesothelioma. Eur J Cardiothorac Surg 2018; 53: 960-966.

232 Nelson DB, Rice DC, Niu J, et al. Long-term survival outcomes of cancer-directed surgery for malignant pleural mesothelioma: propensity score matching analysis. J Clin Oncol 2017; 35: 3354-3362.

233 Beebe-Dimmer JL, Fryzek JP, Yee CL, et al. Mesothelioma in the United States: a Surveillance, Epidemiology, and End Results (SEER)-Medicare investigation of treatment patterns and overall survival. Clin Epidemiol 2016; 8: $743-750$.

234 Damhuis RA, Khakwani A, De Schutter H, et al. Treatment patterns and survival analysis in 9014 patients with malignant pleural mesothelioma from Belgium, the Netherlands and England. Lung Cancer 2015; 89: 212-217.

235 Rosskamp M, Macq G, Nackaerts K, et al. Real-life treatment practice for malignant pleural mesothelioma in Belgium. Lung Cancer 2018; 125: 258-264.

236 Treasure T, Lang-Lazdunski L, Waller D, et al. Extra-pleural pneumonectomy versus no extra-pleural pneumonectomy for patients with malignant pleural mesothelioma: clinical outcomes of the Mesothelioma and Radical Surgery (MARS) randomised feasibility study. Lancet Oncol 2011; 12: 763-772.

237 Bovolato P, Casadio C, Billé A, et al. Does surgery improve survival of patients with malignant pleural mesothelioma?: a multicenter retrospective analysis of 1365 consecutive patients. J Thorac Oncol 2014; 9: 390-396.

238 Kucukoner M, Ali Kaplan M, Inal A, et al. Clinical characteristics, treatment and survival outcomes in malignant pleural mesothelioma: an institutional experience in Turkey. J BUON 2014; 19: 164-170.

239 Lim E. A feasibility study comparing (extended) pleurectomy decortication versus no pleurectomy decortication in the multimodality management of patients with malignant pleural mesothelioma: the MARS 2 study. Lung Cancer 2016; 91: Suppl. 1, S71.

240 MacLeod N, Chalmers A, O’Rourke N, et al. Is radiotherapy useful for treating pain in mesothelioma?: a phase II trial. J Thorac Oncol 2015; 10: 944-950.

241 Rich SE, Chow R, Raman S, et al. Update of the systematic review of palliative radiation therapy fractionation for bone metastases. Radiother Oncol 2018; 126: 547-557.

242 Lutz S, Balboni T, Jones J, et al. Palliative radiation therapy for bone metastases: update of an ASTRO Evidence-Based Guideline. Pract Radiat Oncol 2017; 7: 4-12.

243 McDonald R, Ding K, Brundage M, et al. Effect of radiotherapy on painful bone metastases: a secondary analysis of the NCIC Clinical Trials Group Symptom Control Trial SC.23. JAMA Oncol 2017; 3: 953-959.

244 Boutin C, Rey F, Viallat JR. Prevention of malignant seeding after invasive diagnostic procedures in patients with pleural mesothelioma. A randomized trial of local radiotherapy. Chest 1995; 108: 754-758.

245 O'Rourke N, Garcia JC, Paul J, et al. A randomised controlled trial of intervention site radiotherapy in malignant pleural mesothelioma. Radiother Oncol 2007; 84: 18-22.

246 Muirhead R, O'Rourke N. Drain site radiotherapy in malignant pleural mesothelioma: a wasted resource. Eur Respir J 2007; 30: 1021

247 Bydder S, Phillips M, Joseph DJ, et al. A randomised trial of single-dose radiotherapy to prevent procedure tract metastasis by malignant mesothelioma. Br J Cancer 2004; 91: 9-10.

248 Chapman E, Berenstein EG, Diéguez M, et al. Radiotherapy for malignant pleural mesothelioma. Cochrane Database Syst Rev 2006: CD003880.

249 Clive AO, Taylor H, Dobson L, et al. Prophylactic radiotherapy for the prevention of procedure-tract metastases after surgical and large-bore pleural procedures in malignant pleural mesothelioma (SMART): a multicentre, open-label, phase 3, randomised controlled trial. Lancet Oncol 2016; 17: 1094-1104.

250 Bayman N, Appel W, Ashcroft L, et al. Prophylactic irradiation of tracts in patients with pleural mesothelioma: an open-label, multicentre, phase III randomized trial. J Clin Oncol 2019; 37: 1200-1208.

251 Stahel RA, Riesterer O, Xyrafas A, et al. Neoadjuvant (chemo)therapy and extrapleural pneumonectomy of malignant pleural mesothelioma with or without hemithoracic radiotherapy (SAKK 17/04): a randomised, international, multicentre phase 2 trial. Lancet Oncol 2015; 16: 1651-1658.

252 Cho BC, Feld R, Leighl N, et al. A feasibility study evaluating Surgery for Mesothelioma After Radiation Therapy: the "SMART" approach for resectable malignant pleural mesothelioma. J Thorac Oncol 2014; 9: 397-402.

253 Minatel E, Trovo M, Bearz A, et al. Radical radiation therapy after lung-sparing surgery for malignant pleural mesothelioma: survival, pattern of failure, and prognostic factors. Int J Radiat Oncol Biol Phys 2015; 93: 606-613.

254 Rimner A, Zauderer MG, Gomez DR, et al. Phase II Study of Hemithoracic Intensity-Modulated Pleural Radiation Therapy (IMPRINT) as part of lung-sparing multimodality therapy in patients with malignant pleural mesothelioma. J Clin Oncol 2016; 34: 2761-2768.

255 Buikhuisen WA, Hiddinga BI, Baas P, et al. Second line therapy in malignant pleural mesothelioma: a systematic review. Lung Cancer 2015; 89: 223-231.

256 Laurie SA, Gupta A, Chu Q, et al. Brief report: a phase II study of sunitinib in malignant pleural mesothelioma. The NCIC Clinical Trials Group. I Thorac Oncol 2011; 6: 1950-1954.

257 Buikhuisen WA, Burgers JA, Vincent AD, et al. Thalidomide versus active supportive care for maintenance in patients with malignant mesothelioma after first-line (chemo)therapy (NVALT 5): an open-label, multicentre, randomised phase 3 study. Lancet Oncol 2013; 14: 543-551.

258 Buikhuisen WA, Scharpfenecker M, Griffioen AW, et al. A randomized phase II study adding axitinib to pemetrexed-cisplatin in patients with malignant pleural mesothelioma: a single-center trial combining clinical and translational outcomes. J Thorac Oncol 2016; 11: 758-768.

259 Dubey S, Jänne PA, Krug L, et al. A phase II study of sorafenib in malignant mesothelioma: results of Cancer and Leukemia Group B 30307. J Thorac Oncol 2010; 5: 1655-1661.

260 Fennell DA, McDowell C, Busacca S, et al. Phase II clinical trial of first or second-line treatment with bortezomib in patients with malignant pleural mesothelioma. J Thorac Oncol 2012; 7: 1466-1470. 
261 Hassan R, Kindler HL, Jahan T, et al. Phase II clinical trial of amatuximab, a chimeric antimesothelin antibody with pemetrexed and cisplatin in advanced unresectable pleural mesothelioma. Clin Cancer Res 2014; 20: 5927-5936.

262 Jahan T, Gu L, Kratzke R, et al. Vatalanib in malignant mesothelioma: a phase II trial by the Cancer and Leukemia Group B (CALGB 30107). Lung Cancer 2012; 76: 393-396.

263 Krug LM, Wozniak AJ, Kindler HL, et al. Randomized phase II trial of pemetrexed/cisplatin with or without CBP501 in patients with advanced malignant pleural mesothelioma. Lung Cancer 2014; 85: 429-434.

264 O’Brien ME, Gaafar RM, Popat S, et al. Phase II study of first-line bortezomib and cisplatin in malignant pleura mesothelioma and prospective validation of progression free survival rate as a primary end-point for mesothelioma clinical trials (European Organisation for Research and Treatment of Cancer 08052). Eur J Cancer 2013; 49: 2815-2822.

265 Ralli M, Tourkantonis I, Makrilia N, et al. Docetaxel plus gemcitabine as first-line treatment in malignant pleural mesothelioma: a single institution phase II study. Anticancer Res 2009; 29: 3441-3444.

266 Arrieta Ó, Medina LA, Estrada-Lobato E, et al. First-line (chemo)therapy with liposomal doxorubicin plus cisplatin for patients with advanced malignant pleural mesothelioma: phase II trial. Br J Cancer 2012; 106: 1027-1032.

267 Habib EE, Fahmy ES. Chemotherapy management of malignant pleural mesothelioma: a phase II study comparing two popular (chemo)therapy regimens. Clin Transl Oncol 2013; 15: 965-968.

268 Katirtzoglou N, Gkiozos I, Makrilia N, et al. Carboplatin plus pemetrexed as first-line treatment of patients with malignant pleural mesothelioma: a phase II study. Clin Lung Cancer 2010; 11: 30-35.

269 Kovac V, Zwitter M, Rajer M, et al. A phase II trial of low-dose gemcitabine in a prolonged infusion and cisplatin for malignant pleural mesothelioma. Anticancer Drugs 2012; 23: 230-238.

270 Kuribayashi K, Miyata S, Fukuoka K, et al. Methotrexate and gemcitabine combination (chemo)therapy for the treatment of malignant pleural mesothelioma. Mol Clin Oncol 2013; 1: 639-642.

271 Zalcman G, Mazieres J, Margery J, et al. Bevacizumab for newly diagnosed pleural mesothelioma in the Mesothelioma Avastin Cisplatin Pemetrexed Study (MAPS): a randomised, controlled, open-label, phase 3 trial. [Erratum appears in Lancet 2016; 387: e24. PMID: 27115822]. Lancet 2016; 387: 1405-1414.

272 Ceresoli GL, Zucali PA, Mencoboni M, et al. Phase II study of pemetrexed and carboplatin plus bevacizumab as first-line therapy in malignant pleural mesothelioma. Br J Cancer 2013; 109: 552-558.

273 Dowell JE, Dunphy FR, Taub RN, et al. A multicenter phase II study of cisplatin, pemetrexed, and bevacizumab in patients with advanced malignant mesothelioma. Lung Cancer 2012; 77: 567-571.

274 Kindler HL, Karrison TG, Gandara DR, et al. Multicenter, double-blind, placebo-controlled, randomized phase II trial of gemcitabine/cisplatin plus bevacizumab or placebo in patients with malignant mesothelioma. J Clin Oncol 2012; 30: 2509-2515.

275 Nowak AK, Brown C, Millward MJ, et al. A phase II clinical trial of the vascular disrupting agent BNC105P as second line (chemo)therapy for advanced Malignant Pleural Mesothelioma. Lung Cancer 2013; 81: 422-427.

276 Ou SH, Moon J, Garland LL, et al. SWOG S0722: phase II study of mTOR inhibitor everolimus (RAD001) in advanced malignant pleural mesothelioma (MPM). J Thorac Oncol 2015; 10: 387-391.

277 Papa S, Popat S, Shah R, et al. Phase 2 study of sorafenib in malignant mesothelioma previously treated with platinum-containing (chemo)therapy. J Thorac Oncol 2013; 8: 783-787.

278 Wheatley-Price P, Chu Q, Bonomi M, et al. A Phase II Study of PF-03446962 in Patients with Advanced Malignant Pleural Mesothelioma. CCTG Trial IND.207. J Thorac Oncol 2016; 11: 2018-2021.

279 Campbell NP, Kunnavakkam R, Leighl N, et al. Cediranib in patients with malignant mesothelioma: a phase II trial of the University of Chicago Phase II Consortium. Lung Cancer 2012; 78: 76-80.

280 Dudek AZ, Pang H, Kratzke RA, et al. Phase II study of dasatinib in patients with previously treated malignant mesothelioma (cancer and leukemia group B 30601): a brief report. J Thorac Oncol 2012; 7: 755-759.

281 Garland LL, Chansky K, Wozniak AJ, et al. Phase II study of cediranib in patients with malignant pleural mesothelioma: SWOG S0509. J Thorac Oncol 2011; 6: 1938-1945.

282 Ramalingam SS, Belani CP, Ruel C, et al. Phase II study of belinostat (PXD101), a histone deacetylase inhibitor, for second line therapy of advanced malignant pleural mesothelioma. J Thorac Oncol 2009; 4: 97-101.

283 Scherpereel A, Berghmans T, Lafitte JJ, et al. Valproate-doxorubicin: promising therapy for progressing mesothelioma. A phase II study. Eur Respir J 2011; 37: 129-135.

284 Krug LM, Kindler HL, Calvert H, et al. Vorinostat in patients with advanced malignant pleural mesothelioma who have progressed on previous (chemo)therapy (VANTAGE-014): a phase 3, double-blind, randomised, placebo-controlled trial. Lancet Oncol 2015; 16: 447-456.

285 Stebbing J, Powles T, McPherson K, et al. The efficacy and safety of weekly vinorelbine in relapsed malignant pleural mesothelioma. Lung Cancer 2009; 63: 94-97.

286 Tourkantonis I, Makrilia N, Ralli M, et al. Phase II study of gemcitabine plus docetaxel as second-line treatment in malignant pleural mesothelioma: a single institution study. Am J Clin Oncol 2011; 34: 38-42.

287 Calabrò L, Morra A, Fonsatti E, et al. Tremelimumab for patients with (chemo)therapy-resistant advanced malignant mesothelioma: an open-label, single-arm, phase 2 trial. Lancet Oncol 2013; 14: 1104-1111.

288 Calabrò L, Morra A, Fonsatti E, et al. Efficacy and safety of an intensified schedule of tremelimumab for (chemo) therapy-resistant malignant mesothelioma: an open-label, single-arm, phase 2 study. Lancet Respir Med 2015; 3: 301-309.

289 Maio M, Scherpereel A, Calabrò L, et al. Tremelimumab as second-line or third-line treatment in relapsed malignant mesothelioma (DETERMINE): a multicentre, international, randomised, double-blind, placebo-controlled phase 2b trial. Lancet Oncol 2017; 18: 1261-1273.

290 Gregorc V, Zucali PA, Santoro A, et al. Phase II study of asparagine-glycine-arginine-human tumor necrosis factor $\alpha$, a selective vascular targeting agent, in previously treated patients with malignant pleural mesothelioma. J Clin Oncol 2010; 28: 2604-2611.

291 Scagliotti GV, Gaafar R, Nowak A, et al. Nintedanib in combination with pemetrexed/cisplatin for (chemo) therapy-naïve patients with advanced malignant pleural mesothelioma (LUME-Meso): a double-blind, randomised, placebo-controlled phase 3 trial. Lancet Respir Med 2019; 7: 569-580.

292 Grosso F, Steele N, Novello S, et al. Nintedanib plus pemetrexed/cisplatin in patients with malignant pleural mesothelioma: phase II results from the randomized, placebo-controlled LUME-Meso trial. J Clin Oncol 2017; 35: 3591-3600. 

patients with merlin-stratified pleural mesothelioma: COMMAND - a double-blind, randomized, phase II study. J Clin Oncol 2019; 37: 790-798.

294 Szlosarek PW, Steele JP, Nolan L, et al. Arginine deprivation with pegylated arginine deiminase in patients with argininosuccinate synthetase 1-deficient malignant pleural mesothelioma: a randomized clinical trial. JAMA Oncol 2017; 3: 58-66.

295 Alley EW, Lopez J, Santoro A, et al. Clinical safety and activity of pembrolizumab in patients with malignant pleural mesothelioma (KEYNOTE-028): preliminary results from a non-randomised, open-label, phase $1 \mathrm{~b}$ trial. Lancet Oncol 2017; 18: 623-630.

296 Forde PM, Scherpereel A, Tsao AS. Use of immune checkpoint inhibitors in mesothelioma. Curr Treat Options Oncol 2019; 20: 18.

297 Scherpereel A, Mazieres J, Greillier L, et al. Nivolumab or nivolumab plus ipilimumab in malignant pleural mesothelioma patients with relapsed malignant pleural mesothelioma (IFCT-1501 MAPS2): a multicentre, open-label, randomised, non-comparative phase 2 trial. Lancet Oncol 2019; 20: 239-253.

298 Disselhorst M, Quispel-Janssen J, Lalezari F, et al. Ipilimumab and nivolumab in the treatment of recurrent malignant pleural mesothelioma: a phase II study. Lancet Respir Med 2019; 7: 260-270.

299 Calabrò L, Morra A, Giannarelli D, et al. Tremelimumab combined with durvalumab in patients with mesothelioma (NIBIT-MESO-1): an open-label, non-randomised, phase 2 study. Lancet Respir Med 2018; 6: 451-460.

300 Nowak AK, Lesterhuis WJ, Hughes BGM, et al. DREAM: a phase II study of durvalumab with first line (chemo) therapy in mesothelioma - first results. J Clin Oncol 2018; 36: Suppl. 15, 8503-8503.

301 Süveg K, Putora PM, Berghmans T, et al. Current efforts in research of pleural mesothelioma - an analysis of the ClinicalTrials.gov registry. Lung Cancer 2018; 124: 12-18.

302 Armato SG 3rd, Nowak AK. Revised modified response evaluation criteria in solid tumors for assessment of response in malignant pleural mesothelioma (version 1.1). J Thorac Oncol 2018; 13: 1012-1021.

303 Abdel-Rahman O, Elsayed Z, Mohamed H, et al. Radical multimodality therapy for malignant pleural mesothelioma. Cochrane Database Syst Rev 2018; 1: CD012605.

304 De Bondt C, Psallidas I, Van Schil PEY, et al. Combined modality treatment in mesothelioma: a systemic literature review with treatment recommendations. Transl Lung Cancer Res 2018; 7: 562-573.

305 Travis WD, Brambilla E, Burke AP, et al. WHO Classification Tumours of the Lung and Pleura. 4th Edn. Lyon, IARC Press, 2015.

306 Righi L, Duregon E, Vatrano S, et al. BRCA1-Associated Protein 1 (BAP1) immunohistochemical expression as a diagnostic tool in malignant pleural mesothelioma classification: a large retrospective study. J Thorac Oncol 2016; 11: 2006-2017.

307 Bibby AC, Dorn P, Psallidas I, et al. ERS/EACTS statement on the management of malignant pleural effusions. Eur Respir J 2018; 52: 1800349.

308 O'Brien ME, Watkins D, Ryan C, et al. A randomised trial in malignant mesothelioma (M) of early (E) versus delayed (D) (chemo)therapy in symptomatically stable patients: the MED trial. Ann Oncol 2006; 17: 270-275.

309 Jackson MB, Pounder D, Price C, et al. Percutaneous cervical cordotomy for the control of pain in patients with pleural mesothelioma. Thorax 1999; 54: 238-241.

310 Girgis S, Smith A, Lambert S, et al. "It sort of hit me like a baseball bat between the eyes": a qualitative study of the psychosocial experiences of mesothelioma patients and carers. Support Care Cancer 2019; 27: 631-638.

311 Robinson BW, Lake RA. Advances in malignant mesothelioma. N Engl J Med 2005; 353: 1591-1603.

312 Muers MF, Stephens RJ, Fisher P, et al. Active symptom control with or without (chemo)therapy in the treatment of patients with malignant pleural mesothelioma (MS01): a multicentre randomised trial. Lancet 2008; 371: 1685-1694.

313 Bibby AC, Tsim S, Kanellakis N, et al. Malignant pleural mesothelioma: an update on investigation, diagnosis and treatment. Eur Respir Rev 2016; 25: 472-486.

314 Vogelzang NJ, Rusthoven JJ, Symanowski J, et al. Phase III study of pemetrexed in combination with cisplatin versus cisplatin alone in patients with malignant pleural mesothelioma. J Clin Oncol 2003; 21: 2636-2644.

315 Doyle C. Combination immunotherapy superior to monotherapy in patients with melanoma. Am Health Drug Benefits 2015; 8: 41

316 Tan A, Porcher R, Crequit P, et al. Differences in treatment effect size between overall survival and progression-free survival in immunotherapy trials: a meta-epidemiologic study of trials with results posted at clinicaltrials.gov. J Clin Oncol 2017; 35: 1686-1694.

317 Tumeh PC, Harview CL, Yearley JH, et al. PD-1 blockade induces responses by inhibiting adaptive immune resistance. Nature 2014; 515: 568-571

318 Gao J, Shi LZ, Zhao H, et al. Loss of IFN- $\gamma$ pathway genes in tumor cells as a mechanism of resistance to anti-CTLA-4 therapy. Cell 2016; 167: 397-404.

319 Thapa B, Salcedo A, Lin X, et al. The immune microenvironment, genome-wide copy number aberrations, and survival in mesothelioma. J Thorac Oncol 2017; 12: 850-859.

320 Rizvi NA, Hellmann MD, Snyder A, et al. Cancer immunology. Mutational landscape determines sensitivity to PD-1 blockade in non-small cell lung cancer. Science 2015; 348: 124-128.

321 Postow MA, Callahan MK, Barker CA, et al. Immunologic correlates of the abscopal effect in a patient with melanoma. N Engl J Med 2012; 366: 925-931.

322 Serrels A, Lund T, Serrels B, et al. Nuclear FAK controls chemokine transcription, Tregs, and evasion of anti-tumor immunity. Cell 2015; 163: 160-173.

323 Voron T, Colussi O, Marcheteau E, et al. VEGF-A modulates expression of inhibitory checkpoints on CD8+ T cells in tumors. J Exp Med 2015; 212: 139-148.

324 Muller AJ, DuHadaway JB, Donover PS, et al. Inhibition of indoleamine 2,3-dioxygenase, an immunoregulatory target of the cancer suppression gene Bin1, potentiates cancer (chemo)therapy. Nat Med 2005; 11: 312-319.

325 Koyama S, Akbay EA, Li YY, et al. Adaptive resistance to therapeutic PD-1 blockade is associated with upregulation of alternative immune checkpoints. Nat Commun 2016; 7: 10501

326 Szlosarek PW, Klabatsa A, Pallaska A, et al. In vivo loss of expression of argininosuccinate synthetase in malignant pleural mesothelioma is a biomarker for susceptibility to arginine depletion. Clin Cancer Res 2006; 12: 7126-7131. 
Beddowes E, Spicer J, Chan PY, et al. Phase 1 dose-escalation study of pegylated arginine deiminase, cisplatin, and pemetrexed in patients with argininosuccinate synthetase 1-deficient thoracic cancers. J Clin Oncol 2017; 35: $1778-1785$.

328 LaFave LM, Béguelin W, Koche R, et al. Loss of BAP1 function leads to EZH2-dependent transformation. Nat Med 2015; 21: 1344-1349.

329 Bononi A, Giorgi C, Patergnani S, et al. BAP1 regulates IP3R3-mediated $\mathrm{Ca}^{2+}$ flux to mitochondria suppressing cell transformation. Nature 2017; 546: 549-553.

330 Guazzelli A, Meysami P, Bakker E, et al. BAP1 status determines the sensitivity of malignant mesothelioma cells to gemcitabine treatment. Int J Mol Sci 2019; 20: E429.

331 van Zandwijk N, Pavlakis N, Kao SC, et al. Safety and activity of microRNA-loaded minicells in patients with recurrent malignant pleural mesothelioma: a first-in-man, phase 1, open-label, dose-escalation study. Lancet Oncol 2017; 18: 1386-1396.

332 Bononi A, Yang H, Giorgi C, et al. Germline BAP1 mutations induce a Warburg effect. Cell Death Differ 2017; 24: 1694-1704. 